\title{
Buckling and nonlinear dynamics of elastically coupled double-beam systems
}

\author{
Ivana Bochicchio ${ }^{\mathrm{a}, *}$, Claudio Giorgi ${ }^{\mathrm{b}}$, Elena Vuk ${ }^{\mathrm{b}}$ \\ a DipMat, Università degli studi di Salerno, Italy \\ ${ }^{\mathrm{b}}$ DICATAM, Università degli studi di Brescia, Italy
}

\section{A R T I C L E I N F O}

\section{Article history:}

Received 9 December 2015

Received in revised form

17 June 2016

Accepted 26 June 2016

Available online 29 June 2016

MSC:

35B41

$35 \mathrm{G} 50$

$35 \mathrm{Q} 74$

$74 \mathrm{~B} 20$

$74 \mathrm{G} 60$

$74 \mathrm{H} 40$

74K10

\section{Keywords:}

Double-beam system

Steady states

Buckling

Nonlinear oscillations

Global attractor

\begin{abstract}
A B S T R A C T
This paper deals with damped transverse vibrations of elastically coupled double-beam system under even compressive axial loading. Each beam is assumed to be elastic, extensible and supported at the ends. The related stationary problem is proved to admit both unimodal (only one eigenfunction is involved) and bimodal (two eigenfunctions are involved) buckled solutions, and their number depends on structural parameters and applied axial loads. The occurrence of a so complex structure of the steady states motivates a global analysis of the longtime dynamics. In this regard, we are able to prove the existence of a global regular attractor of solutions. When a finite set of stationary solutions occurs, it consists of the unstable manifolds connecting them.
\end{abstract}

(c) 2016 Elsevier Ltd. All rights reserved.

\section{Introduction}

In this paper we investigate the properties of damped transverse vibrations and dynamical buckling of a coupled doublebeam system under even compressive axial loading. The system models a sandwich structure with an elastic filler. It is composed of two equal WK-beams (according to the nonlinear model of Woinowsky-Krieger [37]), which are connected by linear springs and simply supported at the ends (see Fig. 1).

The in-plane dynamics is ruled by the following nonlinear system:

$$
\left\{\begin{array}{c}
\partial_{t t} u_{1}+\delta \partial_{x x x x} u_{1}+\nu \partial_{t} u_{1}-\left(\ell+\gamma\left\|\partial_{x} u_{1}\right\|_{L^{2}(0, L)}^{2}\right) \partial_{x x} u_{1} \\
+\kappa\left[u_{1}-u_{2}\right]=f_{1}, \\
\partial_{t t} u_{2}+\delta \partial_{x x x x} u_{2}+\nu \partial_{t} u_{2}-\left(\ell+\gamma\left\|\partial_{x} u_{2}\right\|_{L^{2}(0, L)}^{2}\right) \partial_{x x} u_{2} \\
-\kappa\left[u_{1}-u_{2}\right]=f_{2},
\end{array}\right.
$$

\footnotetext{
* Corresponding author.

E-mail address: ibochicchio@unisa.it (I. Bochicchio).
}

where the unknown variables $u_{i}:[0, L] \times \mathbb{R}^{+} \rightarrow \mathbb{R}(i=1,2)$ represent the downward deflections in the vertical plane of the midline of the beams with respect to their reference configuration at rest. Both beams are hinged at their ends, so that

$u_{i}(0, t)=u_{i}(L, t)=\partial_{x x} u_{i}(0, t)=\partial_{x x} u_{i}(L, t)=0, \quad t \in[0, \infty), \quad i=1,2$.

The unknown fields are required to satisfy the following initial conditions:

$u_{i}(x, 0)=u_{i}^{0}(x), \quad \partial_{t} u_{i}(x, 0)=v_{i}^{0}(x), \quad x \in[0, L], \quad i=1,2$,

where $u_{1}^{0}, u_{2}^{0}, v_{1}^{0}$ and $v_{2}^{0}$ are given functions which fulfill (2). The WKbeams are assumed to have equal length $L$ and unitary mass. In the reference (natural) configuration they are straight and parallel, and their spacing is $d$. They are connected by linear elastic springs with common stiffness $\kappa$ and free length $d$. Sources $f_{i}, i=1,2$, represent the given vertical load distributions. The positive constants $\delta$ and $\nu$ denote the flexural rigidity of the beams and the viscosity of the external environment, respectively. Finally, $\gamma$ is a positive constant, whereas the parameter $\ell$ summarizes the effect of the axial force acting at one end of each beam and is positive when both beams are stretched, negative when compressed. 


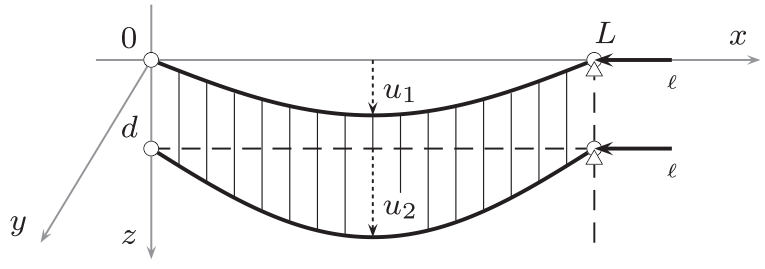

Fig. 1. In-plane oscillations of a double-beam sandwich system.

A derivation of the WK-beam model within a thermoelastic framework can be found in [18]. According to the modeling approach devised therein, the WK-beam equation is a simplification of the nonlinear von Kármán one-dimensional model, where longitudinal (horizontal in our case) displacements are condensed by integrating the corresponding equation in which longitudinal inertia is neglected. Unlike the usual Euler-Bernoulli linear theory, a nonlinear but uniform term accounting for extensibility of the beam is retained into the equation of the transversal vibration. We stress that all material constants in (1) are dimensionless (see [18] for details). In particular

$\delta=\frac{h^{2}}{6 L^{2}}>0, \quad \ell=\frac{2 D}{L} \in \mathbb{R}$,

where $h$ and $D$ are the thickness of the beam and its longitudinal displacement at the ends, respectively. As usual, both are assumed to be considerably shorter than the length $L$. Finally, we remark that the elongation of the coupling springs must take account of the horizontal displacements of their anchor points. Nevertheless, in (1) the strain in the springs is approached by the difference between the vertical displacements of the two beams. This may be accounted for assuming the maximum horizontal displacement, $|D|$, to be negligible if compared with the reference spacing of the beams, namely $|D| \ll d$.

System (1) may be also used to describe out-of-plane oscillations, both vertical and torsional, of a girder bridge where the road bed is modeled by an elastic rug connecting two lateral WK-beams (see Fig. 2). In this case, however, the lateral movements of the beams are neglected by the model.

Although all results obtained hereafter apply to both material models, for the sake of definiteness we shall refer to the former, only. In addition, due to the recasting of the problem into an abstract setting, we stress that the present analysis can be easily extended to (Berger) plate-type sandwich structures with hinged and normally loaded boundaries. In spite of a relatively wide literature concerning statics and dynamics of a single WK-beam (see e.g. $[2,3,5,7,8,14,15,19,22,31,37]$ and references therein), we are not aware of analytic studies which consider the elastic coupling of two or more nonlinear beams of this type. On the other hand, mathematical models of sandwich beam-type and plate-type structures raised a wide interest in the literature, due to their relevance in many branches of modern civil, mechanical and aerospace engineering. In particular in the 80 s the phenomenon of nonlinear buckling mode interaction stimulated much interest and has been investigated by many authors. In particular we recall the fundamental contribution by Budiansky [11] and the many technical papers by Sridharan (see for instance $[4,32]$ ). Recently, after

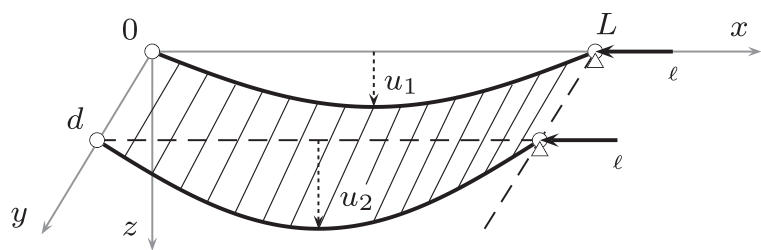

Fig. 2. Out-of-plane oscillations of a double-beam girder bridge. some pioneer works (see, for instance, $[23,29]$ ) concerning interaction buckling between two beams, a lot of papers deal with mechanical properties of axially loaded and elastically connected linear double beam systems (see, for instance, [21,25,26, $35,36,38,39])$.

The aim of this paper is to give a contribution on this subject by scrutinizing statics and dynamics of the initial boundary value problem (1)-(3). Its novelty and relevance relies on the complete characterization of the long time behavior, which emphasizes the different behavior of the nonlinear system (1) with respect to double-beam linear systems previously considered. It is worth noting that in a wider context concerning the localization of vibration modes and buckling patterns [24,27,30], nonlinearities play the same role than imperfections do in linear systems.

The occurrence of a very complex structure of the steady states motivates a global analysis of the longtime dynamics of system (1) (Sections 4 and 5). In this regard, due to the dissipative nature of the system $(\nu>0)$, we are able to prove the existence of a global regular attractor of solutions. In particular, when a finite set of stationary solutions occurs, the global attractor is given by the union of the unstable manifolds connecting them (Section 5.3).

\section{Preliminary results}

Introducing a suitable functional framework, we recast the original system (1) into an abstract setting. Let $(H,\langle\cdot, \cdot\rangle,\|\cdot\|)$ be a real Hilbert space, and let $A: \mathcal{D}(A) \Subset H \rightarrow H$ be a strictly positive selfadjoint operator, whose distinct eigenvalues and eigenfunctions are $\lambda_{i}>0$ and $\psi_{i}, i \in \mathbb{N}$, respectively. For $\tau \in \mathbb{R}$, we introduce the Hilbert spaces

$H_{\tau}=\mathcal{D}\left(A^{\tau / 4}\right), \quad\langle u, v\rangle_{\tau}=\left\langle A^{\tau / 4} u, A^{\tau / 4} v\right\rangle, \quad\|u\|_{\tau}=\left\|A^{\tau / 4} u\right\|$.

The symbol $\langle\cdot, \cdot\rangle$ will also be used to denote the duality product between $H_{\tau}$ and its dual space $H_{-\tau}$. In particular, we have the compact embeddings $H_{\tau+1} \Subset H_{\tau}$, along with the generalized Poincaré inequalities

$\lambda_{1}\|w\|_{\tau}^{4} \leq\|w\|_{\tau+1}^{4}, \quad \forall w \in H_{\tau+1}$,

and we define the family of product Hilbert spaces

$\mathcal{H}_{\tau}=H_{\tau+2} \times H_{\tau} \times H_{\tau+2} \times H_{\tau}, \quad \tau \in[0,2]$.

In all these notations the index $\tau$ is omitted when $\tau=0$. Then, we state on $\mathcal{H}$ the following abstract Cauchy problem:

$\left\{\begin{array}{l}\partial_{t t} u_{1}+\delta A u_{1}+\nu \partial_{t} u_{1}+\left(\ell+\gamma\left\|u_{1}\right\|_{1}^{2}\right) A^{1 / 2} u_{1}+\kappa\left(u_{1}-u_{2}\right)=f_{1}, \\ \partial_{t t} u_{2}+\delta A u_{2}+\nu \partial_{t} u_{2}+\left(\ell+\gamma\left\|u_{2}\right\|_{1}^{2}\right) A^{1 / 2} u_{2}-\kappa\left(u_{1}-u_{2}\right)=f_{2},\end{array}\right.$

$\left(u_{1}(0), \partial_{t} u_{1}(0), u_{2}(0), \partial_{t} u_{2}(0)\right)=\left(u_{1}^{0}, v_{1}^{0}, u_{2}^{0}, v_{2}^{0}\right) \in \mathcal{H}$.

The original problem (1)-(3) can be viewed as a special case of (5)-(6) by assuming $A=\partial_{x x x x}$ and $H=L^{2}(0, L)$. We stress that this abstract formulation cannot be applied when boundary conditions differ from (2) (for instance, if clamped-clamped or hingedclamped ends are prescribed). Really, the original coupled system can be described by means of a single operator $A$ only if the beams are assumed to be hinged at their ends. Afterwards, a weak solution of (5)-(6) will be denoted by $\sigma: \mathbb{R}^{+} \rightarrow \mathcal{H}$,

$\sigma(t)=\left(u_{1}(t), \partial_{t} u_{1}(t), u_{2}(t), \partial_{t} u_{2}(t)\right)$.

For further convenience, (5) may be rewritten as a system with a symmetric nonlinear coupling term independent of $\kappa$. Indeed, letting

$w=\frac{1}{2}\left(u_{1}+u_{2}\right), \quad v=\frac{1}{2}\left(u_{1}-u_{2}\right), \quad f=\frac{1}{2}\left(f_{1}+f_{2}\right), \quad g=\frac{1}{2}\left(f_{1}-f_{2}\right)$, 
(5) takes the following form:

$$
\left\{\begin{array}{r}
\partial_{t t} w+\delta A w+\nu \partial_{t} w+\left[\ell+\frac{\gamma}{2}\left(\|w+v\|_{1}^{2}+\|w-v\|_{1}^{2}\right)\right] A^{1 / 2} w \\
+\frac{\gamma}{2}\left(\|w+v\|_{1}^{2}-\|w-v\|_{1}^{2}\right) A^{1 / 2} v=f, \\
\partial_{t t} v+\delta\left(A+2 \frac{\kappa}{\delta} I\right) v+\nu \partial_{t} v+\left[\ell+\frac{\gamma}{2}\left(\|w+v\|_{1}^{2}+\|w-v\|_{1}^{2}\right)\right] A^{1 / 2} v \\
+\frac{\gamma}{2}\left(\|w+v\|_{1}^{2}-\|w-v\|_{1}^{2}\right) A^{1 / 2} w=g .
\end{array}\right.
$$

\subsection{Energy norm and semigroup of solutions}

Henceforth, $c$ will denote a generic positive constant which possibly (but implicitly) depends on the structural constants of the problem. In addition, $Q: \mathbb{R}_{0}^{+} \rightarrow \mathbb{R}^{+}$will denote a generic increasing monotone function which explicitly depends only on $R$, but implicitly also depends on the structural constants of the problem. The actual expressions of $c$ and $Q$ may change, even within the same line of a given equation.

The total energy of a solution $\sigma$ is defined as

$$
\begin{aligned}
E(\sigma(t))= & \mathcal{E}(\sigma(t))+\frac{1}{2 \gamma}\left[\left(\ell+\gamma\left\|u_{1}(t)\right\|_{1}^{2}\right)^{2}+\left(\ell+\gamma\left\|u_{2}(t)\right\|_{1}^{2}\right)^{2}\right] \\
& +\kappa\left\|u_{1}(t)-u_{2}(t)\right\|^{2},
\end{aligned}
$$

where $\mathcal{E}$ represents the energy norm of $\sigma$ in $\mathcal{H}$, namely

$\mathcal{E}(\sigma(t))=\|\sigma(t)\|_{\mathcal{H}}^{2}=\delta\left(\left\|u_{1}(t)\right\|_{2}^{2}+\left\|u_{2}(t)\right\|_{2}^{2}\right)+\left\|\partial_{t} u_{1}(t)\right\|^{2}+\left\|\partial_{t} u_{2}(t)\right\|^{2}$.

After assuming that a continuous solution $\sigma(t), t \geq 0$, does exist, suitable identities and energy estimates are obtained. They all are written here in a formal setting and could be made rigorous with the use of mollifiers (a procedure which involves boring and routine calculations). The very same identities and estimates apply to the Galerkin approximation scheme which is required to prove existence.

The energy identity is (formally) obtained after multiplying (5) by $\partial_{t} u_{1}$ and $(5)_{2}$ by $\partial_{t} u_{2}$. It reads

$$
\begin{aligned}
& \frac{d}{d t} E(\sigma(t))+2 \nu\left(\left\|\partial_{t} u_{1}(t)\right\|^{2}+\left\|\partial_{t} u_{2}(t)\right\|^{2}\right) \\
& \quad=2\left(\left\langle\partial_{t} u_{1}(t), f_{1}\right\rangle+\left\langle\partial_{t} u_{2}(t), f_{2}\right\rangle\right) .
\end{aligned}
$$

Lemma 1. Let $f_{i} \in H_{-2}, i=1,2$, and $\ell \in \mathbb{R}, \gamma, \delta, \nu, \kappa>0$. For all $t>0$ and initial data $z=\left(u_{1}^{0}, v_{1}^{0}, u_{2}^{0}, v_{2}^{0}\right) \in \mathcal{H}$ with $\|z\|_{\mathcal{H}} \leq R$, we have

$\mathcal{E}(\sigma(t)) \leq Q(R)$.

Proof. We introduce the functional $\mathcal{L}: \mathcal{H} \rightarrow \mathbb{R}$ given by

$\mathcal{L}(\sigma)=E(\sigma)-2\left(\left\langle u_{1}, f_{1}\right\rangle+\left\langle u_{2}, f_{2}\right\rangle\right)$.

Along any solution $\sigma(t)$ to (5), the time function $\mathcal{L}(\sigma(t))$ is nonincreasing. Actually, from the energy identity (8) it follows

$\frac{d}{d t} \mathcal{L}(\sigma(t))=-2 \nu\left(\left\|\partial_{t} u_{1}(t)\right\|^{2}+\left\|\partial_{t} u_{2}(t)\right\|^{2}\right) \leq 0$,

which ensures that

$\mathcal{L}(\sigma(t)) \leq \mathcal{L}(z) \leq Q(R)$,

for all $z \in \mathcal{H}$ with $\|z\|_{\mathcal{H}} \leq R$. Since $E \geq \mathcal{E}$, from (9) we obtain the estimate

$\mathcal{L} \geq \mathcal{E}-2\left(\left\langle u_{1}, f_{1}\right\rangle+\left\langle u_{2}, f_{2}\right\rangle\right) \geq \frac{1}{2} \mathcal{E}-2\left(\left\|f_{1}\right\|_{-2}^{2}+\left\|f_{2}\right\|_{-2}^{2}\right)$,

which finally yields

$\mathcal{E}(\sigma(t)) \leq 2 \mathcal{L}(\sigma(t))+4\left(\left\|f_{1}\right\|_{-2}^{2}+\left\|f_{2}\right\|_{-2}^{2}\right) \leq Q(R)$.

Lemma 2. Let $f_{i} \in H_{-2}, i=1,2$, and $\ell \in \mathbb{R}, \gamma, \delta, \nu, \kappa>0$. For all initial data $z \in \mathcal{H}$, the abstract Cauchy problem (5)-(6) admits a unique solution $\sigma \in C(0, T ; \mathcal{H})$, which continuously depends on the initial data.

The proof is omitted in that it can be obtained by paralleling Proposition 1 in [9], and relies on a standard Faedo-Galerkin approximation scheme (see $[2,3]$ ) together with a slight generalization of the usual Gronwall lemma. In particular, the uniform-intime estimates needed to prove the global existence are exactly the same as in Lemma 1.

According to Lemma 2, system (5)-(6) generates a strongly continuous semigroup (or dynamical system) $S(t)$ on $\mathcal{H}$, i.e. for a given initial data $z \in \mathcal{H}$,

$\sigma(t)=S(t) z, \quad \mathcal{E}(t)=\|S(t) z\|_{\mathcal{H}}^{2}$.

Moreover, $S(t)$ admits a Lyapunov functional, according to the following result.

Lemma 3. If $f_{i} \in H_{-2}, i=1,2$, then $\mathcal{L}$, defined in (9) is a Lyapunov functional for $S(t)$, that is a function $\mathcal{L} \in C(\mathcal{H}, \mathbb{R})$ satisfying the following conditions:

(i) $\mathcal{L}(z) \rightarrow \infty$ if and only if $\|z\|_{\mathcal{H}} \rightarrow \infty$;

(ii) $\mathcal{L}(S(t) z)$ is nonincreasing for any $z \in \mathcal{H}$;

(iii) $\mathcal{L}(S(t) z)=\mathcal{L}(z)$ for all $t>0$ implies that $z \in \mathcal{S}$, where

$\mathcal{S}=\{z \in \mathcal{H}: S(t) z=z, \forall t>0\}$

is the set of all stationary solutions.

Proof. By the continuity of $\mathcal{L}$, and by means of the estimates

$\frac{1}{2} E(z)-c \leq \mathcal{L}(z) \leq \frac{3}{2} E(z)+c, \quad \forall z \in \mathcal{H}$,

assertion (i) can be easily proved. The nonincreasing monotonicity of $\mathcal{L}$, along the trajectories departing from $z$, namely (ii), has been yet shown in the proof of Lemma 1 . Finally, if $\mathcal{L}$ is constant in time, from (10) we have $\partial_{t} u_{1}(t)=\partial_{t} u_{2}(t)=0$ for all $t>0$, which implies that $u_{1}(t)=\bar{u}_{1}, u_{2}(t)=\bar{u}_{2}$ are constants and that they satisfy (1). Hence, $z=S(t) z=\left(\bar{u}_{1}, 0, \bar{u}_{2}, 0\right)$ for all $t>0$, and then $z \in \mathcal{S}$.

\section{Steady states}

In this section we analyze the stationary problem related to (5) in the homogeneous case $\left(f_{1}=f_{2} \equiv 0\right)$. Depending on the model parameters $\kappa$ and $\ell$, there exist nontrivial (buckled) solutions, both unimodal and bimodal. In spite of the perfect symmetry of the system, for special values of the parameters we obtain non-symmetric static solutions where the elastic energy is not evenly distributed between the two beams. The proof of these results can be found in [16] and is summarized in the Appendix. The nonhomogeneous case can be handled according to [15].

Let $\mathcal{S}_{0}$ be the set of stationary solutions to the homogeneous problem. It is made of vectors $z_{0}=\left(u_{1}, 0, u_{2}, 0\right) \in \mathcal{H}$ such that $\left(u_{1}, u_{2}\right) \in H_{2} \times H_{2}$ are weak solutions to the system

$\left\{\begin{array}{l}A u_{1}+C_{1}\left(\left\|u_{1}\right\|_{1}\right) A^{1 / 2} u_{1}+k\left(u_{1}-u_{2}\right)=0, \\ A u_{2}+C_{2}\left(\left\|u_{2}\right\|_{1}\right) A^{1 / 2} u_{2}-k\left(u_{1}-u_{2}\right)=0,\end{array}\right.$

where

$C_{i}\left(\left\|u_{i}\right\|_{1}\right)=\left(\ell+\gamma\left\|u_{i}\right\|_{1}^{2}\right) / \delta, \quad k=\kappa / \delta, \quad i=1,2$.

In particular, it reduces to

$\left\{\begin{array}{l}u_{1}^{\prime \prime \prime \prime}-C_{1} u_{1}^{\prime \prime}+k\left(u_{1}-u_{2}\right)=0, \\ u_{2}^{\prime \prime \prime}-C_{2} u_{2}^{\prime \prime}-k\left(u_{1}-u_{2}\right)=0, \\ u_{i}(0)=u_{i}(L)=u_{i}^{\prime \prime}(0)=u_{i}^{\prime \prime}(L)=0, \quad i=1,2,\end{array}\right.$ 
when $A=\partial_{x x x x}$ and $\lambda_{n}=n^{4} \pi^{4} / L^{4}, \psi_{n}(x)=\sin (n \pi x / L)$. Of course, the trivial solution $u_{1}=u_{2}=0$ does always exist.

\subsection{Unimodal solutions}

First, we look for solutions where each field involves only one eigenfunction, namely we consider

$u_{1}(x)=a_{n} \psi_{n}(x), \quad u_{2}(x)=b_{m} \psi_{m}(x), \quad m, n \in \mathbb{N}$.

After replacing these expressions, from (12) we achieve the system

$\left\{\begin{array}{l}\left(\lambda_{n}+C_{1} \sqrt{\lambda_{n}}+k\right) a_{n} \psi_{n}-k b_{m} \psi_{m}=0, \\ \left(\lambda_{m}+C_{2} \sqrt{\lambda_{m}}+k\right) b_{m} \psi_{m}-k a_{n} \psi_{n}=0,\end{array}\right.$

where

$C_{1}=\left(\ell+\frac{L}{2} \gamma a_{n}^{2} \sqrt{\lambda_{n}}\right) / \delta, \quad C_{2}=\left(\ell+\frac{L}{2} \gamma b_{m}^{2} \sqrt{\lambda_{m}}\right) / \delta$.

When $m \neq n$, it easily follows that $a_{n}=b_{m}=0$ and $u_{1}=u_{2}=0$. Accordingly, if a nontrivial solution $\left(u_{1}, u_{2}\right)$ of this kind does exist then it is unimodal, in the sense that both $u_{1}$ and $u_{2}$ should involve the same eigenfunction,

$u_{1}(x)=a_{n} \psi_{n}(x), \quad u_{2}(x)=b_{n} \psi_{n}(x), \quad n \in \mathbb{N}$.

In the sequel, we consider a fixed $n \in \mathbb{N}$.

Theorem 1. Depending on the values of $\ell \in \mathbb{R}$, there exist at most nine unimodal solutions to (11) involving the $n$-th eigenfunction of $A$. In particular:

- when $\ell \geq-\delta \sqrt{\lambda_{n}}$ there exists only the null solution $\left(a_{n}^{(0)}, b_{n}^{(0)}\right)=(0,0)$;

- at $\ell=-\delta \sqrt{\lambda_{n}}$ the null solution bifurcates into a couple of inphase solutions,

$\left(a_{n}^{(1)}, b_{n}^{(1)}\right)=\left( \pm \alpha_{n}, \pm \alpha_{n}\right)$;

- at $\ell=-\delta \sqrt{\lambda_{n}}-2 k / \sqrt{\lambda_{n}}$ the null solution bifurcates into a couple of out-of-phase solutions,

$\left(a_{n}^{(2)}, b_{n}^{(2)}\right)=\left( \pm \beta_{n}, \mp \beta_{n}\right)$,

- at $\ell=-\delta \sqrt{\lambda_{n}}-3 k / \sqrt{\lambda_{n}}$ each out-of-phase solution bifurcates into a couple of solutions with unequally distributed energy, namely

$$
\left(a_{n}^{(3)}, b_{n}^{(3)}\right)=\left( \pm \omega_{n}^{+}, \mp \omega_{n}^{-}\right), \quad\left(a_{n}^{(4)}, b_{n}^{(4)}\right)=\left( \pm \omega_{n}^{-}, \mp \omega_{n}^{+}\right) .
$$

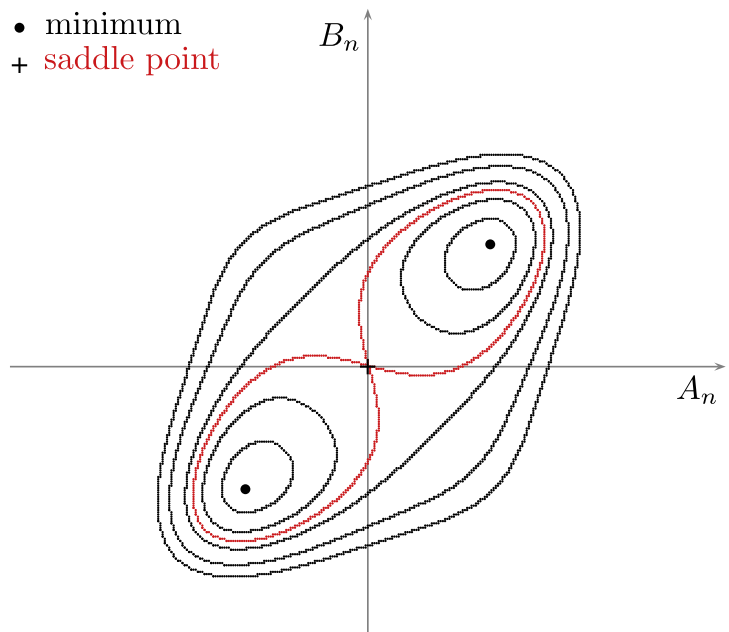

Summarizing, there are

- one solution (the null solution) in the range $\ell \mid \delta \geq-\sqrt{\lambda_{n}}$;

- three solutions in the range $-\sqrt{\lambda_{n}}-2 k /\left(\delta \sqrt{\lambda_{n}}\right) \leq \ell \mid \delta<-\sqrt{\lambda_{n}}$;

- five solutions in the range $-\sqrt{\lambda_{n}}-3 k /\left(\delta \sqrt{\lambda_{n}}\right) \leq \ell / \delta<-\sqrt{\lambda_{n}}-2 k /\left(\delta \sqrt{\lambda_{n}}\right)$;

- nine solutions in the range $\ell / \delta<-\sqrt{\lambda_{n}}-3 k /\left(\delta \sqrt{\lambda_{n}}\right)$.

Remark 1. This situation occurs also in the study of buckling for a rectangular plate under in-plane loading. This phenomenon is named mode jumping: its buckling pattern exhibits a symmetric breaking (secondary) bifurcation, that is the symmetry group of the equations remains unchanged, but the solutions which bifurcate break the symmetry (see [34, pp. 149, 242]).

By replacing (14) into the expression of the total energy $E$, we get

$$
\begin{aligned}
E\left(a_{n}, b_{n}\right)= & \lambda_{n}^{2} \delta\left(a_{n}^{2}+b_{n}^{2}\right)+\frac{1}{2}\left(\frac{\ell}{\gamma}+\lambda_{n} a_{n}^{2}\right)^{2}+\frac{1}{2}\left(\frac{\ell}{\gamma}+\lambda_{n} b_{n}^{2}\right)^{2} \\
& +\kappa\left(a_{n}-b_{n}\right)^{2}
\end{aligned}
$$

and using the level-set representation we infer that (see Figs. 3 and 4)

- in the range $\ell \geq-\delta \sqrt{\lambda_{n}}$ the null solution is the unique minimum of $E$;

- in the range $-\delta \sqrt{\lambda_{n}}-2 k / \sqrt{\lambda_{n}} \leq \ell<-\delta \sqrt{\lambda_{n}}$ the energy $E$ admits two minima and a saddle point (the null solution);

- in the range $-\delta \sqrt{\lambda_{n}}-3 k / \sqrt{\lambda_{n}} \leq \ell<-\delta \sqrt{\lambda_{n}}-2 k / \sqrt{\lambda_{n}}$ the energy $E$ admits two minima, two saddle points and a maximum (the null solution);

- in the range $\ell<-\delta \sqrt{\lambda_{n}}-3 k / \sqrt{\lambda_{n}}$ the energy $E$ admits four minima, four saddle points and a maximum (the null solution).

If we restrict our attention to the amplitude $a_{n}$ of $u_{1}$, Fig. 5 shows the corresponding bifurcation diagram depending on the values of $q_{n}=-\ell-\delta \sqrt{\lambda_{n}}$.

Remark 2. After the secondary bifurcation, the double-beam system exhibits steady-states where the beams have different elastic energies. Such non-symmetric equilibria correspond to unstable branches and cannot be observed. In spite of this, their occurrence is relevant in the longtime global dynamic of the two beams, for it may lead up to non-symmetric energy exchanges

$$
\begin{aligned}
& \text { - maximum } \\
& \text { - minimum } \\
& + \text { saddle point }
\end{aligned}
$$

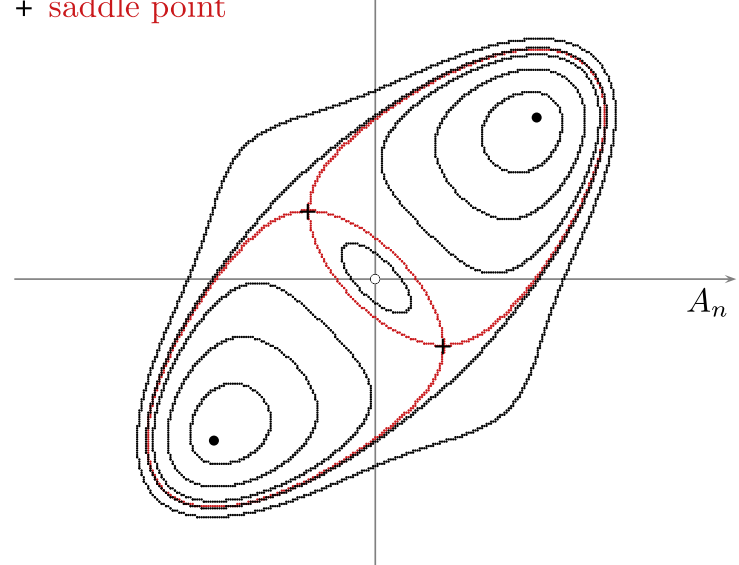

Fig. 3. The energy level sets of $E$ at $\ell=-2.5,-3.5\left(\gamma=\delta=\kappa=\lambda_{n}=1\right)$. 


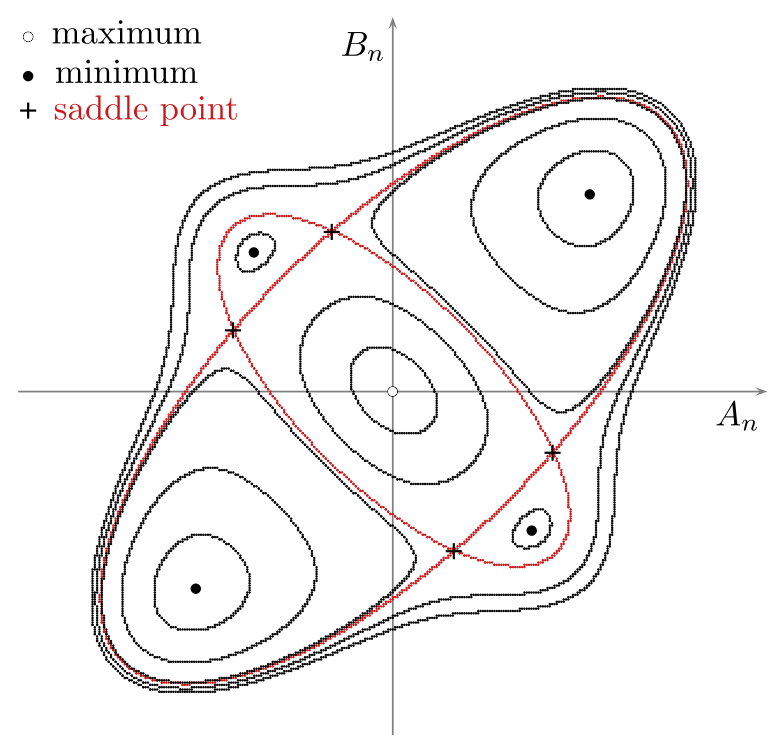

Fig. 4. The energy level sets of $E$ at $\ell=-5\left(\gamma=\delta=\kappa=\lambda_{n}=1\right)$.

between them.

\subsection{Bimodal solutions}

It is well known that all the steady states of a single extensible beam are expressed as unimodal functions (see, for instance $[7,15])$. We scrutinize here whether this is the case even for the double-beam system under consideration. At a first attempt, we are looking for bimodal solutions. Of course, if solutions of this kind do exist, they cannot take the in-phase shape because of the system uncoupling. A more general approach is devised in [16].

\subsubsection{The resonant set}

Let

$\mu_{n}=\delta \sqrt{\lambda_{n}}, \quad \nu_{n}(\kappa)=\mu_{n}+2 \kappa / \sqrt{\lambda_{n}}, \quad n \in \mathbb{N}$,

which represent the eigenvalues of the operators $\delta A^{1 / 2}$ and $\delta A^{1 / 2}+2 \kappa A^{-1 / 2}$, respectively. According to the previous section, system (12) exhibits unimodal in-phase bifurcation when $\ell=-\mu_{n}$ and unimodal out-of-phase bifurcation when $\ell=-\nu_{n}(\kappa)$.

Without any loss of generality, we assume $m, n \in \mathbb{N}, m>n$. Then $\mu_{m}>\mu_{n}$, but $\nu_{n}(\kappa)$ could overlap either $\mu_{\mathrm{m}}$ or $\nu_{m}(\kappa)$ if $\kappa$ is properly chosen. In the former case,

$\nu_{n}\left(\kappa_{m n}^{\star}\right)=\mu_{m} \quad \Longleftrightarrow \quad \kappa_{m n}^{\star}=\frac{\delta}{2}\left[\sqrt{\lambda_{n} \lambda_{m}}-\lambda_{n}\right]$.

In the latter case,

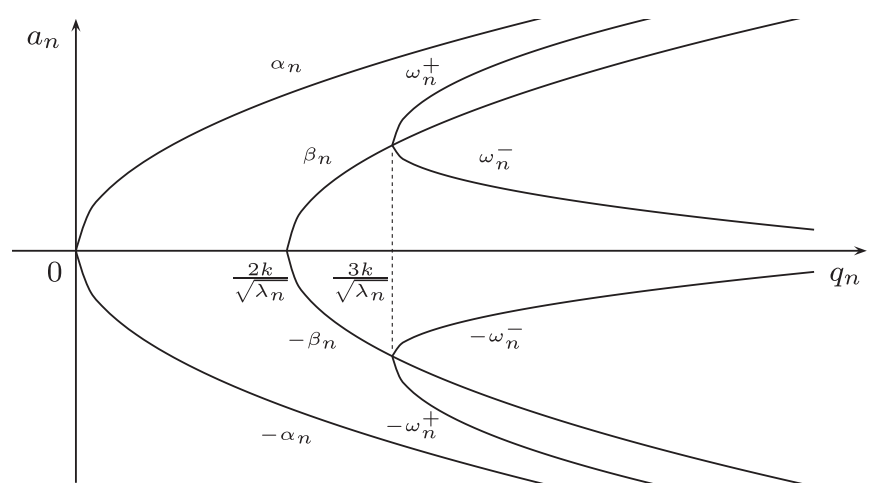

Fig. 5. The buckled solutions $a_{n}^{(i)}, i=1,2,3,4$, for a fixed $n \in \mathbb{N}$. $\nu_{n}\left(\kappa_{m n}^{\dagger}\right)=\nu_{m}\left(\kappa_{m n}^{\dagger}\right) \quad \Longleftrightarrow \quad \kappa_{m n}^{\dagger}=\frac{\delta}{2} \sqrt{\lambda_{n} \lambda_{m}}$

and then $\nu_{n}\left(\kappa_{m n}^{\dagger}\right)=\mu_{n}+\mu_{m}$. The set $\mathcal{R}$ of all the values of $\kappa$ that produce some overlapping is named resonant set:

$\mathcal{R}=\left\{\kappa \in \mathbb{R}^{+}\right.$: either $\kappa=\kappa_{m n}^{\dagger}$ or $\kappa=\kappa_{m n}^{\star}$, for some integer $\left.m>n\right\}$.

Now, we look for nontrivial bimodal solutions of the form

$u_{1}(x)=a_{n} \psi_{n}(x)+c_{m} \psi_{m}(x), \quad u_{2}(x)=b_{n} \psi_{n}(x)+d_{m} \psi_{m}(x)$,

where $a_{n}, b_{n}, c_{m}, d_{m} \neq 0$. When both beams have the same elastic energy, the following result holds (see the Appendix).

Theorem 2. Given $m>n$, bimodal solutions such that $\left\|u_{1}\right\|_{1}=\left\|u_{2}\right\|_{1}$ are allowed only if $\kappa \in \mathcal{R}$. In particular,

- when $\kappa=\kappa_{m n}^{\dagger}$ there exist $\infty^{1}$ bimodal solutions, where both modes are out-of-phase, provided that $\ell<-\left(\mu_{n}+\mu_{m}\right)$;

- when $\kappa=\kappa_{m n}^{\star}$ there exist $\infty^{1}$ bimodal solutions, where the m-mode is in-phase and the n-mode is out-of-phase, provided that $\ell<-\mu_{m}$.

It is apparent that after bifurcation infinitely many $(n, m)$ bimodal solutions just occur instead of $n$ and $m$ unimodal ones when their critical values $\nu_{n}$ and $\nu_{m}$ overlap.

A different result holds if the total elastic energy at equilibrium are unequally distributed among the beams. After introducing $\zeta_{m n}=\sqrt{\lambda_{m} / \lambda_{n}}>1$ and

$\phi_{m n}(\kappa)=\frac{\left(\zeta_{m n}+1\right)+\left(\zeta_{m n}-1\right)\left(1-2 \kappa_{m n}^{\dagger} / \kappa\right)^{2}}{\zeta_{m n}\left(1-2 \kappa_{m n}^{\dagger} / \kappa\right)}$,

$\psi_{m n}(\kappa)=\frac{\left(\zeta_{m n}+1\right)-\left(\zeta_{m n}-1\right)\left(1-2 \kappa_{m n}^{\dagger} / \kappa\right)^{2}}{1-2 \kappa_{m n}^{\dagger} / \kappa}$,

we are able to prove (see the Appendix).

Theorem 3. Given $m>n$, four distinct bimodal solutions such that $\left\|u_{1}\right\|_{1} \neq\left\|u_{2}\right\|_{1}$ exist if and only if either

$\kappa_{m n}^{\dagger}<\kappa<2 \kappa_{m n}^{\dagger}$, and $\ell_{1}^{*}(\kappa)<\ell<\ell_{2}^{*}(\kappa)$

or

$\kappa<\kappa_{m n}^{\star}$, and $\ell<\ell_{1}^{*}(\kappa)$,

where

$\ell_{1}^{*}(\kappa)=-\nu_{n}(\kappa)+\kappa \frac{1+\phi_{m n}(\kappa)}{\sqrt{\lambda_{n}}}, \quad \ell_{2}^{*}(\kappa)=-\nu_{m}(\kappa)+\kappa \frac{1+\psi_{m n}(\kappa)}{\sqrt{\lambda_{m}}}$.

\section{The exponential decay}

Within this section, we restrict our attention to the homogeneous system, namely we assume $f_{i}=0$ into (5). It is easy to check that the linear part of the differential operator acting on $u_{i}$ fulfills the following lemma.

Lemma 4. (See $\left[6\right.$, Lemma 4.5]) Let $\mathcal{F}_{\ell}\left(u_{i}\right)=\delta A u_{i}+\ell A^{1 / 2} u_{i}(i=1,2)$ and

$C_{\ell}= \begin{cases}1 & \text { if } \ell \geq 0, \\ \left(1+\frac{\ell}{\delta \sqrt{\lambda_{1}}}\right) & \text { if }-\delta \sqrt{\lambda_{1}}<\ell<0 .\end{cases}$

Then 
$\left\langle\mathcal{F}_{\ell}\left(u_{i}\right), u_{i}\right\rangle \geq \delta C_{\ell}\left\|u_{i}\right\|_{2}^{2}$,

provided that $\ell>-\delta \sqrt{\lambda_{1}}$.

As a consequence, letting $S_{0}(t)$ be the semigroup which is generated by (5) when $f_{i}=0$, we are able to prove the following result.

Theorem 4. Let $z \in \mathcal{H},\|z\|_{\mathcal{H}} \leq R$. Provided that $\ell>-\delta \sqrt{\lambda_{1}}$, all solutions $S_{0}(t) z$ decay exponentially, i.e. there exists a positive constant $c$ such that

$\mathcal{E}(t) \leq Q(R) e^{-c t}$.

Proof. We introduce the functional

$\Phi=E+\varepsilon\left\langle u_{1}, \partial_{t} u_{1}\right\rangle+\varepsilon\left\langle u_{2}, \partial_{t} u_{2}\right\rangle-\frac{1}{\gamma} \ell^{2}$,

and we choose

$\varepsilon=\min \left\{1, \nu, \delta \lambda_{1} C_{\ell}, \frac{2 \delta \lambda_{1} C_{\ell}}{\nu}\right\}$

Taking into account (17), this constant is positive provided that $\ell>-\delta \sqrt{\lambda_{1}}$. After recalling the definition of $E$, we remark that

$$
\begin{aligned}
\Phi= & \left\langle\mathcal{F}_{\ell}\left(u_{1}\right), u_{1}\right\rangle+\left\langle\mathcal{F}_{\ell}\left(u_{2}\right), u_{2}\right\rangle+\left\|\partial_{t} u_{1}\right\|^{2}+\left\|\partial_{t} u_{2}\right\|^{2} \\
& +\frac{1}{2} \gamma\left(\left\|u_{1}\right\|_{1}^{4}+\left\|u_{2}\right\|_{1}^{4}\right)+\varepsilon\left\langle u_{1}, \partial_{t} u_{1}\right\rangle+\varepsilon\left\langle u_{2}, \partial_{t} u_{2}\right\rangle \\
& +\kappa\left\|u_{1}-u_{2}\right\|^{2} .
\end{aligned}
$$
is

The first step is to prove the equivalence between $\mathcal{E}$ and $\Phi$, that

$\frac{C_{\ell}}{2} \mathcal{E} \leq \Phi \leq Q(R) \mathcal{E}$

Indeed, a lower bound is provided by virtue of (19) and Lemma 4,

$\Phi \geq\left(1-\frac{\varepsilon}{2}\right)\left(\left\|\partial_{t} u_{1}\right\|^{2}+\left\|\partial_{t} u_{2}\right\|^{2}\right)+\left(\delta C_{\ell}-\frac{\varepsilon}{2 \lambda_{1}}\right)\left(\left\|u_{1}\right\|_{2}^{2}+\left\|u_{2}\right\|_{2}^{2}\right)$

$\geq \frac{C_{\ell}}{2} \varepsilon$.

On the other hand, by applying Young inequality and Lemma 1 tailored to $S_{0}(t)$, the upper bound of $\Phi$ follows:

$\Phi \leq\left[2+\frac{\kappa}{\lambda_{1} \delta}+\frac{|\ell|}{\delta \sqrt{\lambda_{1}}}+\frac{\varepsilon^{2}}{2 \lambda_{1} \delta}+\frac{\gamma Q(R)}{2 \lambda_{1} \delta^{2}}\right] \mathcal{E}=Q(R) \mathcal{E}$.

The last step is to prove the exponential decay of $\Phi$. To this aim, we obtain the identity

$$
\begin{aligned}
\frac{d}{d t} \Phi & +\varepsilon \Phi+2(\nu-\varepsilon)\left(\left\|\partial_{t} u_{1}\right\|^{2}+\left\|\partial_{t} u_{2}\right\|^{2}\right)+\frac{\varepsilon}{2} \gamma\left(\left\|u_{1}\right\|_{1}^{4}+\left\|u_{2}\right\|_{1}^{4}\right) \\
& +\varepsilon(\nu-\varepsilon)\left(\left\langle\partial_{t} u_{1}, u_{1}\right\rangle+\left\langle\partial_{t} u_{2}, u_{2}\right\rangle\right)=0,
\end{aligned}
$$

where $\varepsilon$ is given by (19). Exploiting (4), (21) and the Young inequality, we have

$$
\begin{aligned}
\frac{d}{d t} \Phi+\varepsilon \Phi+(\nu-\varepsilon)\left(\left\|\partial_{t} u_{1}\right\|^{2}\right. & \left.+\left\|\partial_{t} u_{2}\right\|^{2}\right) \leq \varepsilon^{2} \frac{(\nu-\varepsilon)}{4 \lambda_{1}}\left(\left\|u_{1}\right\|_{2}^{2}+\left\|u_{2}\right\|_{2}^{2}\right) \\
& \leq \frac{\varepsilon^{2} \nu}{2 \delta \lambda_{1} C_{\ell}} \Phi,
\end{aligned}
$$

from which it follows

$\frac{d}{d t} \Phi+\frac{\varepsilon}{2 \delta \lambda_{1} C_{\ell}}\left[2 \delta \lambda_{1} C_{\ell}-\varepsilon \nu\right] \Phi \leq 0$.

Letting $c=\varepsilon\left[2 \delta \lambda_{1} C_{\ell}-\varepsilon \nu\right] / 2 \delta \lambda_{1} C_{\ell}$, which is positive by virtue of (19), we conclude
$\frac{C_{\ell}}{2} \mathcal{E}(t) \leq \Phi(t) \leq \Phi(0) e^{-c t} \leq Q(R) e^{-c t}$

\section{The global attractor}

The existence of a Lyapunov functional (see Lemma 3) ensures that bounded sets of initial data have bounded orbits. In this section, we provide the existence of the global attractor by showing a suitable (really, exponential) asymptotic compactness property of the semigroup. Namely, we prove the following.

Theorem 5. Let $f_{i} \in H_{-2}, i=1,2$, be fixed. Then, the semigroup $S(t)$ acting on $\mathcal{H}$ possesses a connected global attractor $\mathcal{A} \subset \mathcal{H}$. In particular, $\mathcal{A}$ is bounded in $\mathcal{H}_{2}$, so that its regularity is optimal.

By standard arguments of the theory of dynamical systems (see $[1,20,33])$, the first part of Theorem 5 can be established by exploiting the following:

Lemma 5. Assume that $S(t)$ fulfills the asymptotic compactness property. That is, for every $R>0$, there exist a function $\psi_{R}: \mathbb{R}^{+} \rightarrow \mathbb{R}^{+}$ vanishing at infinity and a compact set $\mathcal{K}_{R} \subset \mathcal{H}$ such that the semigroup $S(t)$ can be split into the sum $L(t)+K(t)$, where the oneparameter operators $L(t)$ and $K(t)$ fulfill

$\|L(t) z\|_{\mathcal{H}} \leq \psi_{R}(t), \quad K(t) z \in \mathcal{K}_{R}$,

whenever $\|z\|_{\mathcal{H}} \leq R$ and $t \geq 0$. Then, $S(t)$ possesses a connected global attractor $\mathcal{A} \subset \mathcal{K}_{R_{0}}$ for some $R_{0}>0$.

According to the scheme first devised in [19], we split the solution $S(t) z$ into the sum

$S(t) z=L(t) z+K(t) z$

where

$L(t) z=\left(u_{1}^{L}(t), \partial_{t} u_{1}^{L}(t), u_{2}^{L}(t), \partial_{t} u_{2}^{L}(t)\right)$

$K(t) z=\left(u_{1}^{K}(t), \partial_{t} u_{1}^{K}(t), u_{2}^{K}(t), \partial_{t} u_{2}^{K}(t)\right)$

respectively solve the systems

$\left\{\begin{array}{l}\partial_{t t} u_{1}^{L}+\delta A u_{1}^{L}+\nu \partial_{t} u_{1}^{L}+\left(\ell+\gamma\left\|u_{1}\right\|_{1}^{2}\right) A^{1 / 2} u_{1}^{L}+a u_{1}^{L}=0 \\ \partial_{t t} u_{2}^{L}+\delta A u_{2}^{L}+\nu \partial_{t} u_{2}^{L}+\left(\ell+\gamma\left\|u_{2}\right\|_{1}^{2}\right) A^{1 / 2} u_{2}^{L}+a u_{2}^{L}=0 \\ \left(u_{1}^{L}(0), \partial_{t} u_{1}^{L}(0), u_{2}^{L}(0), \partial_{t} u_{2}^{L}(0)\right)=z,\end{array}\right.$

$\left\{\begin{array}{l}\partial_{t t} u_{1}^{K}+\delta A u_{1}^{K}+\nu \partial_{t} u_{1}^{K}+\left(\ell+\gamma\left\|u_{1}\right\|_{1}^{2}\right) A^{1 / 2} u_{1}^{K}-a u_{1}^{L}+\kappa\left(u_{1}-u_{2}\right)=f_{1}, \\ \partial_{t t} u_{2}^{K}+\delta A u_{2}^{K}+\nu \partial_{t} u_{2}^{K}+\left(\ell+\gamma\left\|u_{2}\right\|_{1}^{2}\right) A^{1 / 2} u_{2}^{K}-a u_{2}^{L}-\kappa\left(u_{1}-u_{2}\right)=f_{2}, \\ \left(u_{1}^{K}(0), \partial_{t} u_{1}^{K}(0), u_{2}^{K}(0), \partial_{t} u_{2}^{K}(0)\right)=0,\end{array}\right.$

where $a>0$ is large enough that the following inequality holds (see [19])

$\frac{1}{2}\|u\|_{2}^{2} \leq\|u\|_{2}^{2}+\ell\|u\|_{1}^{2}+a\|u\|^{2} \leq m\|u\|_{2}^{2}$

for all $u \in H_{2}$ and for some $m=m(\ell, a) \geq 1$.

The proof of Lemma 5 is carried out in the next two subsections, and the last part of Theorem 5 follows from the compact embedding $\mathcal{H}_{2} \Subset \mathcal{H}$.

\subsection{The exponential decay of $L(t)$}

We prove here the following lemma.

Lemma 6. Let $\|z\|_{\mathcal{H}} \leq R$. There is $c=c(R)>0$ such that $\|L(t) z\|_{\mathcal{H}} \leq Q(R) e^{-c t}$. 
Proof. Let $\mathcal{E}_{0}$ be the energy norm of the one-parameter operator $L$ $(t)$, namely

$$
\begin{aligned}
\mathcal{E}_{0}(L(t) z)\|L(t) z\|_{\mathcal{H}}^{2}= & \delta\left(\left\|u_{1}^{L}(t)\right\|_{2}^{2}+\left\|u_{2}^{L}(t)\right\|_{2}^{2}\right)+\left\|\partial_{t} u_{1}^{L}(t)\right\|^{2} \\
& +\left\|\partial_{t} u_{2}^{L}(t)\right\|^{2} .
\end{aligned}
$$

We introduce the functional

$$
\begin{aligned}
\Phi_{0}(t) & =\Phi_{0}\left(L(t) z, u_{1}(t), u_{2}(t)\right)=\mathcal{E}_{0}(L(t) z) \\
& +\ell\left(\left\|u_{1}^{L}(t)\right\|_{1}^{2}+\left\|u_{2}^{L}(t)\right\|_{1}^{2}\right)+a\left(\left\|u_{1}^{L}(t)\right\|^{2}+\left\|u_{2}^{L}(t)\right\|^{2}\right) \\
& +\gamma\left(\left\|u_{1}(t)\right\|_{1}^{2}\left\|u_{1}^{L}(t)\right\|_{1}^{2}+\left\|u_{2}(t)\right\|_{1}^{2}\left\|u_{2}^{L}(t)\right\|_{1}^{2}\right) \\
& +\varepsilon\left(\left\langle\partial_{t} u_{1}^{L}(t), u_{1}^{L}(t)\right\rangle+\left\langle\partial_{t} u_{2}^{L}(t), u_{2}^{L}(t)\right\rangle\right) .
\end{aligned}
$$

Using Lemma 1 and (24), we obtain the following lower and upper bounds for $\Phi_{0}$ :

$\frac{1}{4} \mathcal{E}_{0} \leq \Phi_{0} \leq Q(R) \mathcal{E}_{0}$.

The time-derivative of $\Phi_{0}$ along a solution to system (22) reads

$$
\begin{aligned}
\frac{d}{d t} \Phi_{0}+\varepsilon \Phi_{0}+2(\nu-\varepsilon)\left(\left\|\partial_{t} u_{1}^{L}\right\|^{2}+\left\|\partial_{t} u_{2}^{L}\right\|^{2}\right) \\
=2 \gamma\left(\left\langle\partial_{t} u_{1}, A^{1 / 2} u_{1}\right\rangle\left\|u_{1}^{L}\right\|_{1}^{2}+\left\langle\partial_{t} u_{2}, A^{1 / 2} u_{2}\right\rangle\left\|u_{2}^{L}\right\|_{1}^{2}\right) \\
-\varepsilon(\nu-\varepsilon)\left(\left\langle u_{1}^{L}, \partial_{t} u_{1}^{L}\right\rangle+\left\langle u_{2}^{L}, \partial_{t} u_{2}^{L}\right\rangle\right),
\end{aligned}
$$

where the second term can be estimated in terms of $\varepsilon_{0}$ as follows:

$$
\begin{aligned}
2 \gamma & \left\langle\partial_{t} u_{i}, A^{1 / 2} u_{i}\right\rangle\left\|u_{i}^{L}\right\|_{1}^{2}-\varepsilon(\nu-\varepsilon)\left\langle u_{i}^{L}, \partial_{t} u_{i}^{L}\right\rangle \\
\leq & \frac{\gamma}{\varepsilon^{2} \sqrt{\lambda_{1}}}\left\|\partial_{t} u_{i}\right\|^{2}\left\|u_{i}^{L}\right\|_{2}^{2}+\frac{\gamma \varepsilon^{2}}{\sqrt{\lambda_{1}}}\left\|A^{1 / 2} u_{i}\right\|^{2}\left\|u_{i}^{L}\right\|_{2}^{2}+(\nu-\varepsilon)\left\|\partial_{t} u_{i}^{L}\right\|^{2} \\
& +\varepsilon^{2}(\nu-\varepsilon)\left\|u_{i}^{L}\right\|^{2} \\
\leq & \frac{1}{\varepsilon^{2}} Q(R)\left\|\partial_{t} u_{i}\right\| \mathcal{E}_{0}+\varepsilon^{2} Q(R) \mathcal{E}_{0}+(\nu-\varepsilon)\left\|\partial_{t} u_{i}^{L}\right\|^{2}+\frac{\varepsilon^{2}(\nu-\varepsilon)}{\lambda_{1}} \mathcal{E}_{0} .
\end{aligned}
$$

Choosing $\varepsilon$ as small as needed in order that $\varepsilon Q(R)+\frac{\varepsilon(\nu-\varepsilon)}{\lambda_{1}}<\frac{1}{8}$, we obtain

$\frac{d}{d t} \Phi_{0}+\varepsilon \Phi_{0} \leq \frac{1}{\varepsilon^{2}} Q(R)\left(\left\|\partial_{t} u_{1}\right\|+\left\|\partial_{t} u_{2}\right\|\right) \mathcal{E}_{0}+\frac{\varepsilon}{8} \mathcal{E}_{0}$

and finally, since $\frac{1}{4} \mathcal{E}_{0} \leq \Phi_{0}$, we can infer

$\frac{d}{d t} \Phi_{0}+\frac{\varepsilon}{2} \Phi_{0} \leq \frac{1}{\varepsilon^{2}} Q(R)\left(\left\|\partial_{t} u_{1}\right\|+\left\|\partial_{t} u_{2}\right\|\right) \Phi_{0}$.

In order to estimate the r.h.s. we need some control on the dissipation of the original semigroup. Hence, by following the procedure of [9, Lemma 6.2] we obtain,

Lemma 7 (Integral control of dissipation). For any $\epsilon>0$ and $t \geq s \geq 0$,

$\int_{s}^{t}\left(\left\|\partial_{t} u_{1}(y)\right\|+\left\|\partial_{t} u_{2}(y)\right\|\right) d y \leq \epsilon(t-s)+Q_{\epsilon}(R)$.

The exponential decay of $\Phi_{0}$ is then entailed by exploiting a generalized Gronwall Lemma (see [12, Lemma 2.1, 13, Lemma 3.7]). Finally, from (26) the desired decay of $\varepsilon_{0}$ follows.

\subsection{The compactness of $\mathcal{K}_{R}$}

Now, we prove the existence of a compact set $\mathcal{K}_{R}$ which contains all orbits $K(t) z,\|z\|_{\mathcal{H}} \leq R, t \geq 0$. This result is achieved by two steps: first the boundedness of these orbits in a more regular space, $\mathcal{H}_{2}$, is proved in Lemma 8 , then the compactness of $\mathcal{K}_{R}$ is ensured by the compact embedding $\mathcal{H}_{2} \Subset \mathcal{H}$ (see $[9,10]$ ).
Lemma 8. Let $f_{i} \in H_{-2}, i=1,2$, be fixed and let $z \in \mathcal{H},\|z\|_{\mathcal{H}} \leq R$. Then, there exists $K_{R}>0$ such that

$\|K(t) z\|_{\mathcal{H}_{2}} \leq K_{R}, \quad \forall t \geq 0$.

Proof. Let introduce the energy norm of order 2 of $K(t)$, namely $\mathcal{E}^{K}=\delta\left(\left\|u_{1}^{K}\right\|_{4}^{2}+\left\|u_{2}^{K}\right\|_{4}^{2}\right)+\left\|\partial_{t} u_{1}^{K}\right\|_{2}^{2}+\left\|\partial_{t} u_{2}^{K}\right\|_{4}^{2}$.

For any $\varepsilon \in(0,1)$ we define the functional

$J^{K}=\mathcal{E}^{K}+F^{K}+2 \varepsilon\left(\left\langle\partial_{t} u_{1}^{K}, u_{1}^{K}\right\rangle_{2}+\left\langle\partial_{t} u_{2}^{K}, u_{2}^{K}\right\rangle_{2}\right)$,

where

$$
\begin{aligned}
F^{K}= & \left(\ell+\gamma\left\|u_{1}(t)\right\|_{1}^{2}\right)\left\|u_{1}^{K}\right\|_{3}^{2}+\left(\ell+\gamma\left\|u_{2}(t)\right\|_{1}^{2}\right)\left\|u_{2}^{K}\right\|_{3}^{2} \\
& -2\left(\left\langle f_{1}, u_{1}^{K}\right\rangle_{2}+\left\langle f_{2}, u_{2}^{K}\right\rangle_{2}\right) .
\end{aligned}
$$

Choosing $\varepsilon$ small enough, the following bounds hold true:

$\frac{1}{2} \mathcal{E}^{K}-Q(R) \leq J^{K} \leq 2 \mathcal{E}^{K}+Q(R)$.

We estimate the time derivative of $J^{K}$ along a solution $K(t) z$. To this end we first compute

$$
\begin{aligned}
\frac{d}{d t}\left(\mathcal{E}^{K}+F^{K}\right)= & -2 \nu\left(\left\|\partial_{t} u_{1}^{K}\right\|_{2}^{2}+\left\|\partial_{t} u_{2}^{K}\right\|_{2}^{2}\right) \\
& +2 a\left(\left\langle\partial_{t} u_{1}^{K}, u_{1}^{L}\right\rangle_{2}+\left\langle\partial_{t} u_{2}^{K}, u_{2}^{L}\right\rangle_{2}\right) \\
& +2 \gamma\left(\left\|u_{1}^{K}\right\|_{3}^{2}\left\langle u_{1}, \partial_{t} u_{1}\right\rangle_{1}+\left\|u_{2}^{K}\right\|_{3}^{2}\left\langle u_{2}, \partial_{t} u_{2}\right\rangle_{1}\right) \\
& -2 \kappa\left\langle u_{1}-u_{2}, \partial_{t}\left(u_{1}^{K}-u_{2}^{K}\right)\right\rangle_{2} .
\end{aligned}
$$

We deal with each term at the r.h.s. separately. By virtue of the interpolation inequality

$\left|\langle\phi, \psi\rangle_{\tau}\right| \leq\|\phi\|_{\tau+1}\|\psi\|_{\tau-1}$,

taking into account (4) and Lemma 1 , we easily obtain $(i=1,2)$,

$\left\|u_{i}^{K}\right\|_{3}^{2}\left\langle u_{i}, \partial_{t} u_{i}\right\rangle_{1} \leq \frac{1}{\sqrt{\lambda_{1}}}\left\|u_{i}^{K}\right\|_{4}^{2}\left\|u_{i}\right\|_{2}\left\|\partial_{t} u_{i}\right\| \leq Q(R)\left\|\partial_{t} u_{i}\right\| \mathcal{E}^{K}$,

$a\left\langle\partial_{t} u_{i}^{K}, u_{i}^{L}\right\rangle_{2} \leq \frac{c}{\varepsilon}\left\|u_{i}^{L}\right\|_{2}^{2}+\frac{\varepsilon}{16} \varepsilon^{K} \leq \frac{Q(R)}{\varepsilon}+\frac{\varepsilon}{16} \varepsilon^{K}$.

Analogously

$\kappa\left|\left\langle u_{1}-u_{2}, \partial_{t}\left(u_{1}^{K}-u_{2}^{K}\right)\right\rangle_{2}\right| \leq \frac{Q(R)}{\varepsilon}+\frac{\varepsilon}{8} \varepsilon^{K}$.

In addition, a straightforward calculation leads to

$$
\begin{aligned}
& \frac{d}{d t}\left[\left\langle\partial_{t} u_{1}^{K}, u_{1}^{K}\right\rangle_{2}+\left\langle\partial_{t} u_{2}^{K}, u_{2}^{K}\right\rangle_{2}\right] \\
&=\left\|\partial_{t} u_{1}^{K}\right\|_{2}^{2}+\left\|\partial_{t} u_{2}^{K}\right\|_{2}^{2}-\delta\left(\left\|u_{1}^{K}\right\|_{4}^{2}+\left\|u_{2}^{K}\right\|_{4}^{2}\right) \\
&-\nu\left(\left\langle\partial_{t} u_{1}^{K}, u_{1}^{K}\right\rangle_{2}+\left\langle\partial_{t} u_{2}^{K}, u_{2}^{K}\right\rangle_{2}\right)+a\left(\left\langle u_{1}^{K}, u_{1}^{L}\right\rangle_{2}+\left\langle u_{2}^{K}, u_{2}^{L}\right\rangle_{2}\right) \\
&+\left\langle u_{1}^{K}, f_{1}\right\rangle_{2}+\left\langle u_{2}^{K}, f_{2}\right\rangle_{2}-\kappa\left\langle u_{1}-u_{2}, u_{1}^{K}-u_{2}^{K}\right\rangle_{2} \\
&-\left(\ell+\gamma\left\|u_{1}\right\|_{1}^{2}\right)\left\|u_{1}^{K}\right\|_{3}^{2}-\left(\ell+\gamma\left\|u_{2}\right\|_{1}^{2}\right)\left\|u_{2}^{K}\right\|_{3}^{2} .
\end{aligned}
$$

From interpolation inequality (29), we have

$\left|\left\langle u_{1}-u_{2}, u_{1}^{K}-u_{2}^{K}\right\rangle_{2}\right| \leq\left\|u_{1}-u_{2}\right\|_{1}\left\|u_{1}^{K}-u_{2}^{K}\right\|_{3} \leq Q(R) \sqrt{\mathcal{E}^{K}}$,

hence 


$$
\begin{aligned}
& \frac{d}{d t}\left[\left\langle\partial_{t} u_{1}^{K}, u_{1}^{K}\right\rangle_{2}+\left\langle\partial_{t} u_{2}^{K}, u_{2}^{K}\right\rangle_{2}\right] \\
& \quad \leq(1+\nu)\left(\left\|\partial_{t} u_{1}^{K}\right\|_{2}^{2}+\left\|\partial_{t} u_{2}^{K}\right\|_{2}^{2}\right)-\frac{\delta}{2}\left(\left\|u_{1}^{K}\right\|_{4}^{2}+\left\|u_{2}^{K}\right\|_{4}^{2}\right)+Q_{\varepsilon}(R) \\
& \quad+Q(R) \sqrt{\mathcal{E}^{K}} .
\end{aligned}
$$

Collecting all these inequalities we end up with

$$
\begin{gathered}
\frac{d}{d t} J^{K}+2[\nu-\varepsilon(1+\nu)]\left(\left\|\partial_{t} u_{1}^{K}\right\|_{2}^{2}+\left\|\partial_{t} u_{2}^{K}\right\|_{2}^{2}\right)+\varepsilon \delta\left(\left\|u_{1}^{K}\right\|_{4}^{2}+\left\|u_{2}^{K}\right\|_{4}^{2}\right) \\
-\frac{\varepsilon}{2} \varepsilon^{K} \leq Q(R) \sqrt{\mathcal{E}^{K}}+Q(R) \mathcal{E}^{K}\left(\left\|\partial_{t} u_{1}\right\|+\left\|\partial_{t} u_{2}\right\|\right)+Q_{\varepsilon}(R) .
\end{gathered}
$$

Choosing now $\varepsilon=\varepsilon_{0}>0$ small enough, in light of (28) this turns into

$\frac{d}{d t} J^{K}+\frac{\varepsilon_{0}}{2} J^{K} \leq Q(R) \sqrt{J^{K}}+Q(R)\left(\left\|\partial_{t} u_{1}\right\|+\left\|\partial_{t} u_{2}\right\|\right)\left(1+J^{K}\right)+Q(R)$.

Since $J^{K}(0)=0$, once more from Lemma 7 and the generalized Gronwall Lemma (see [12, Lemma 2.1, 13, Lemma 3.7]) we get the thesis.

\subsection{The structure of the attractor}

As well known [1], when a finite set of stationary solutions occurs, the global attractor of the semigroup $S(t)$ consists of the unstable manifolds connecting them. Due to Theorems $1-3$, we can conclude:

Corollary 1. Provided that $\ell \leq-\delta \sqrt{\lambda_{1}}$ and $\kappa \neq \mathcal{R}$, the global attractor $\mathcal{A} \subset \mathcal{H}$ consists of the unstable manifolds connecting the steady states of $S(t)$.

Even if we restrict our attention to unimodal solutions (in this case $\mathcal{H}$ reduces to $\mathbb{R}^{4}$ ), a complete picture of $\mathcal{A}$ is hard to achieve. Really, some informations may be sketched by means of Poincaré sections, which are obtained by intersecting trajectories in the original phase-space with a lower dimensional subspace transversal to the flow of the system. This technique may be exploited for both the damped and undamped $(\nu>0)$ system, although it is a routinary tool of the latter case. Unfortunately, there is no general method to construct a Poincaré map.

Here, we may take advantage of the representation (7) of the system. Indeed, when $f=g=0$ and assuming suitable initial conditions, this system admits special dynamical solutions of the form $(\bar{w}(t), 0)$ and $(0, \bar{v}(t))$, corresponding to perfectly symmetric $\left(\bar{u}_{1}=\bar{u}_{2}\right)$ and antisymmetric $\left(\bar{u}_{1}=-\bar{u}_{2}\right)$ oscillations. If this is the case, then $\bar{w}$ and $\bar{v}$ satisfy the uncoupled first order systems

$$
\begin{gathered}
\left\{\begin{array}{l}
\partial_{t} \bar{\xi}+\delta A \bar{w}+\nu \bar{\xi}+\left[\ell+\gamma\|\bar{w}\|_{1}^{2}\right] A^{1 / 2} \bar{w}=0 \\
\partial_{t} \bar{w}=\bar{\xi}
\end{array}\right. \\
\left\{\begin{array}{l}
\partial_{t} \bar{\eta}+\delta A_{\kappa} \bar{v}+\nu \bar{\eta}+\left[\ell+\gamma\|\bar{v}\|_{1}^{2}\right] A^{1 / 2} \bar{v}=0 \\
\partial_{t} \bar{v}=\bar{\eta}
\end{array}\right.
\end{gathered}
$$

and the phase portraits of their unimodal solutions $\bar{w}(x, t)=\bar{w}_{n}(t) \psi_{n}(x), \quad \bar{v}(x, t)=\bar{v}_{n}(t) \psi_{n}(x), \quad n \in \mathbb{N}$, can be scrutinized as in [31]. For instance, assuming $\frac{\ell}{\delta}<-\sqrt{\lambda_{n}}-3 \frac{k}{\delta \sqrt{\lambda_{n}}}$

we conclude that in each sub-space $\left(w_{n}, \xi_{n}\right)$ and $\left(v_{n}, \eta_{n}\right)$ of the fourdimensional phase-space $\mathcal{H}$ there is a branch of the attractor $\mathcal{A}$, and the basins of attraction of the stable steady-states relying therein may be easily depicted (see Fig. 6).

\section{Conclusions}

In this paper we scrutinized both statics and dynamics of a damped elastically coupled WK double-beam system under even compressive axial loading.

A complete characterization (in closed form) of solutions to the nonlinear stationary problem with vanishing sources is achieved. As well as the null solution, the system may exhibit unimodal (only one eigenfunction is involved) and bimodal (two eigenfunctions are involved) buckled solutions. In Theorem 1 the number of unimodal solutions is proved to depend on the ratios $\ell \mid \delta$ and $\kappa / \delta$. In spite of the perfect symmetry of the system, for special values of the parameters we obtain non-symmetric static solutions where the elastic energy is not evenly distributed between the two beams (see also the Appendix, Fig. 8).

Since the axial displacement $D$ and the thickness $h$ are comparable and considerably shorter than the length $L$ and the spacing $d$ of the beams, under reasonably physical assumptions on the stiffness of the elastic core we may conclude that $|\ell|$ and $\kappa$ share the same order of magnitude $h / L$, whereas $\delta$ is much smaller. Accordingly, the order of magnitude of $|\ell| \delta \mid$ and $\kappa / \delta$ is $L / h \gg 1$. Hence, inequalities on $\lambda_{n}$ in Theorem 1 are not narrowed by the modeling assumptions and all the stationary solutions exhibited here are physically consistent.

The complete analysis of buckling bifurcations (see Fig. 5) and the related picture of the energy level sets (see Figs. 3 and 4) reveal that stability of unimodal stationary solutions strongly depend on the coupling constant $\kappa$. This is not surprising when nonlinear equations are involved. In nonlinear electroelastostatics,

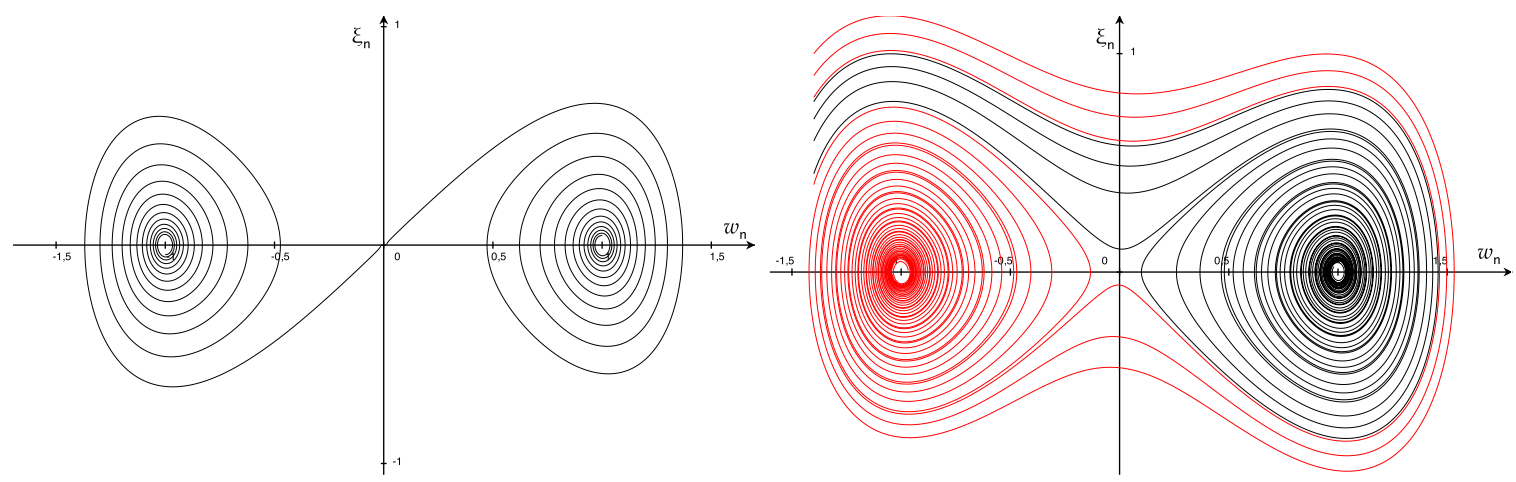

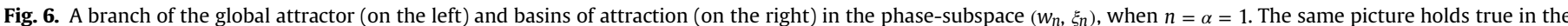
phase-subspace $\left(v_{n}, \eta_{n}\right)$, when $n=\beta=1$ ( $\alpha$ and $\beta$ are the same as in Fig. 7a). 
for instance, the stability of steady states may be crucially dependent on the magnitudes of the electromechanical coupling parameters (see [17]).

The occurrence of a so complex structure of the steady states motivates a global analysis of the longtime damped dynamics. When $-\ell \mid \delta$ is larger than the first eigenvalue $\lambda_{1}$ of the Laplacian operator $A^{1 / 2}$, then in Theorem 4 we prove exponential stability of the (unique) trivial solution. On the contrary, when $\ell \leq-\delta \sqrt{\lambda_{1}}$ buckled steady-states occur and we prove in Theorem 5 the existence of a global regular attractor of dynamical solutions. If in addition $\kappa \neq \mathcal{R}$, the resonant set defined in Section 3.2.1, then there exists a finite number of stationary solutions and the global attractor $\mathcal{A} \subset \mathcal{H}$ consists of the unstable manifolds of trajectories connecting them (see Section 5.3 and figures therein).

\section{Appendix A}

\section{A.1. Proof of Theorem 1}

When $m=n$ from (13) we get the system

$\left\{\begin{array}{l}b_{n}=\omega_{n} a_{n}\left(p_{n}+a_{n}^{2}\right), \\ a_{n}=\omega_{n} b_{n}\left(p_{n}+b_{n}^{2}\right),\end{array}\right.$

where

$p_{n}=\frac{2}{L \gamma \sqrt{\lambda_{n}}}\left(\ell+\delta \sqrt{\lambda_{n}}+\frac{\kappa}{\sqrt{\lambda_{n}}}\right), \quad \omega_{n}=\frac{L \lambda_{n} \gamma}{2 \kappa}$.

Assuming $a_{n}, b_{n} \neq 0$ and putting $Y_{n}=p_{n}+a_{n}^{2}$, from (32) it follows $Y_{n}^{4}-p_{n} Y_{n}^{3}+\frac{p_{n}}{\omega_{n}^{2}} Y_{n}-\frac{1}{\omega_{n}^{4}}=0$.

Accordingly, this equation can be written as a product,

$\left(Y_{n}^{2}-\frac{1}{\omega_{n}^{2}}\right)\left(Y_{n}^{2}-p_{n} Y_{n}+\frac{1}{\omega_{n}^{2}}\right)=0$,

so yielding four solutions

$Y_{n}= \pm 1 / \omega_{n}, \quad Y_{n}=\left(p_{n} \omega_{n} \pm \sqrt{p_{n}^{2} \omega_{n}^{2}-4}\right) / 2 \omega_{n}$.

Letting $q_{n}=-\ell-\delta \sqrt{\lambda_{n}}$ and

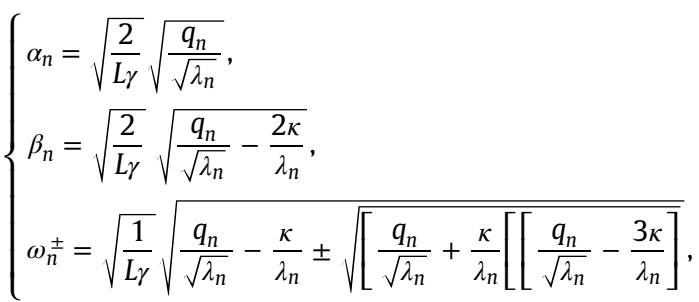

from (32) and (33) we obtain the following expressions for the amplitudes $a_{n}$ and $b_{n}$ :

$\left\{\begin{array}{lll}\left(a_{n}^{(1)}, b_{n}^{(1)}\right)=\left( \pm \alpha_{n}, \pm \alpha_{n}\right) & \text { if } & q_{n}>0, \\ \left(a_{n}^{(2)}, b_{n}^{(2)}\right)=\left( \pm \beta_{n}, \mp \beta_{n}\right) & \text { if } & q_{n}>\frac{2 \kappa}{\sqrt{\lambda_{n}}}, \\ \left(a_{n}^{(3)}, b_{n}^{(3)}\right)=\left( \pm \omega_{n}^{+}, \mp \omega_{n}^{-}\right) & \text {if } & q_{n}>\frac{3 \kappa}{\sqrt{\lambda_{n}}}, \\ \left(a_{n}^{(4)}, b_{n}^{(4)}\right)=\left( \pm \omega_{n}^{-}, \mp \omega_{n}^{+}\right) & \text {if } & q_{n}>\frac{3 \kappa}{\sqrt{\lambda_{n}}},\end{array}\right.$

which provide all real unimodal solutions involving the $n$-th eigenfunction.

When $n=1$ and $\ell<-\delta \sqrt{\lambda_{1}}-3 \kappa / \sqrt{\lambda_{1}}$, these solutions are represented in Figs. 7 and 8 (the subscript 1 is omitted). The buckling modes here depicted resemble a study carried out in [28] within the framework of the "buckling localization".

\section{A.2. Proof of Theorem 2}

From (12) we achieve

$\left\{\begin{array}{l}{\left[\left(\lambda_{n}+C_{1} \sqrt{\lambda_{n}}+k\right) a_{n}-k b_{n}\right] \psi_{n}+\left[\left(\lambda_{m}+C_{1} \sqrt{\lambda_{m}}+k\right) c_{m}-k d_{m}\right] \psi_{m}=0,} \\ {\left[\left(\lambda_{n}+C_{2} \sqrt{\lambda_{n}}+k\right) b_{n}-k a_{n}\left[\psi_{n}+\left[\left(\lambda_{m}+C_{2} \sqrt{\lambda_{m}}+k\right) d_{m}-k c_{m}\right] \psi_{m}=0,\right.\right.}\end{array}\right.$

where

$C_{1}=\frac{\ell}{\delta}+\frac{L \gamma}{2 \delta} X_{1}, \quad X_{1}=a_{n}^{2} \sqrt{\lambda_{n}}+c_{m}^{2} \sqrt{\lambda_{m}}=\frac{2}{L}\left\|u_{1}\right\|_{1}^{2}$,

$C_{2}=\frac{\ell}{\delta}+\frac{L \gamma}{2 \delta} X_{2}, \quad X_{2}=b_{n}^{2} \sqrt{\lambda_{n}}+d_{m}^{2} \sqrt{\lambda_{m}}=\frac{2}{L}\left\|u_{2}\right\|_{1}^{2}$.

Because of the orthogonality of distinct eigenfunctions, we obtain the system

$\left\{\begin{array}{l}k b_{n}=a_{n}\left[\lambda_{n}+k+\sqrt{\lambda_{n}} \ell \mid \delta+\sqrt{\lambda_{n}} L \gamma X_{1} / 2 \delta\right], \\ k d_{m}=c_{m}\left[\lambda_{m}+k+\sqrt{\lambda_{m}} \ell \mid \delta+\sqrt{\lambda_{m}} L \gamma X_{1} / 2 \delta\right], \\ k a_{n}=b_{n}\left[\lambda_{n}+k+\sqrt{\lambda_{n}} \ell \mid \delta+\sqrt{\lambda_{n}} L \gamma X_{2} / 2 \delta\right], \\ k c_{m}=d_{m}\left[\lambda_{m}+k+\sqrt{\lambda_{m}} \ell \mid \delta+\sqrt{\lambda_{m}} L \gamma X_{2} / 2 \delta\right] .\end{array}\right.$

For the sake of simplicity, we let

$\sigma_{j}=\frac{2}{L \gamma}\left(\delta \sqrt{\lambda_{j}}+\frac{\kappa}{\sqrt{\lambda_{j}}}+\ell\right), \quad \xi_{j}=\frac{L \gamma \sqrt{\lambda_{j}}}{2 \kappa}, \quad j=m, n$

and we rewrite the previous system in the compact form

$\left\{\begin{array}{l}b_{n}=\xi_{n} a_{n}\left(\sigma_{n}+X_{1}\right), \\ d_{m}=\xi_{m} c_{m}\left(\sigma_{m}+X_{1}\right), \\ a_{n}=\xi_{n} b_{n}\left(\sigma_{n}+X_{2}\right), \\ c_{m}=\xi_{m} d_{m}\left(\sigma_{m}+X_{2}\right),\end{array}\right.$

from which it follows

$\left\{\begin{array}{l}a_{n}=\xi_{n}^{2} a_{n}\left(\sigma_{n}+X_{1}\right)\left(\sigma_{n}+X_{2}\right), \\ b_{n}=\xi_{n} a_{n}\left(\sigma_{n}+X_{1}\right), \\ c_{m}=\xi_{m}^{2} c_{m}\left(\sigma_{m}+X_{1}\right)\left(\sigma_{m}+X_{2}\right), \\ d_{m}=\xi_{m} c_{m}\left(\sigma_{m}+X_{1}\right) .\end{array}\right.$

From the first and third equations of (36) we obtain a system involving $X_{1}$ and $X_{2}$, only,

$\left\{\begin{array}{l}1=\xi_{n}^{2}\left(\sigma_{n}+X_{1}\right)\left(\sigma_{n}+X_{2}\right), \\ 1=\xi_{m}^{2}\left(\sigma_{m}+X_{1}\right)\left(\sigma_{m}+X_{2}\right) .\end{array}\right.$

If it admits positive solutions $\left(X_{1}^{*}, X_{2}^{*}\right)$, then the four amplitudes of the bimodal solutions $u_{1}$ and $u_{2}$ follow by solving

$\left\{\begin{array}{l}b_{n}=\xi_{n} a_{n}\left(\sigma_{n}+X_{1}^{*}\right), \\ d_{m}=\xi_{m} c_{m}\left(\sigma_{m}+X_{1}^{*}\right), \\ \sqrt{\lambda_{n}} a_{n}^{2}+\sqrt{\lambda_{m}} c_{m}^{2}=X_{1}^{*}, \\ \sqrt{\lambda_{n}} b_{n}^{2}+\sqrt{\lambda_{m}} d_{m}^{2}=X_{2}^{*} .\end{array}\right.$

When the beams have the same elastic energy, system (37) provides

$\left\{\begin{array}{l}X= \pm \xi_{n}^{-1}-\sigma_{n}, \\ X= \pm \xi_{m}^{-1}-\sigma_{m} .\end{array}\right.$

These relations are satisfied provided that 
a)
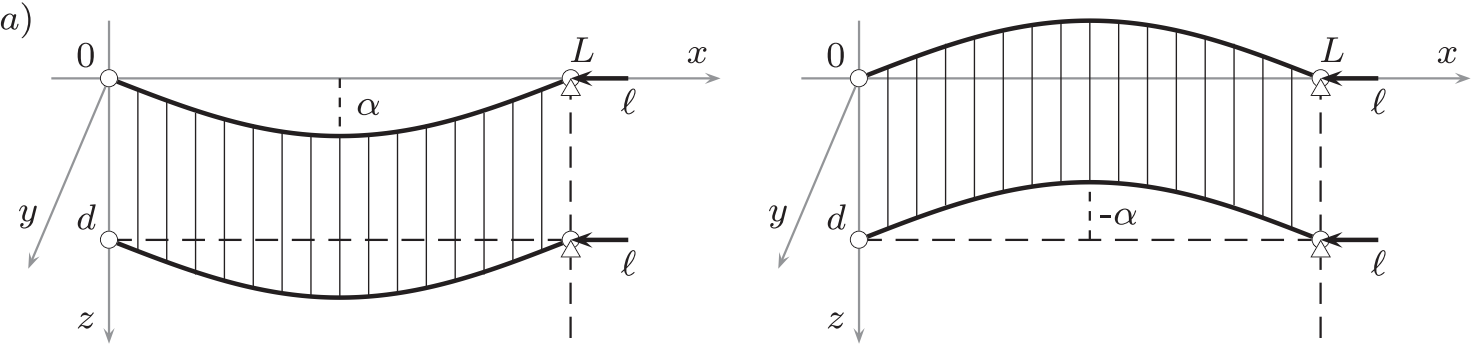

b)
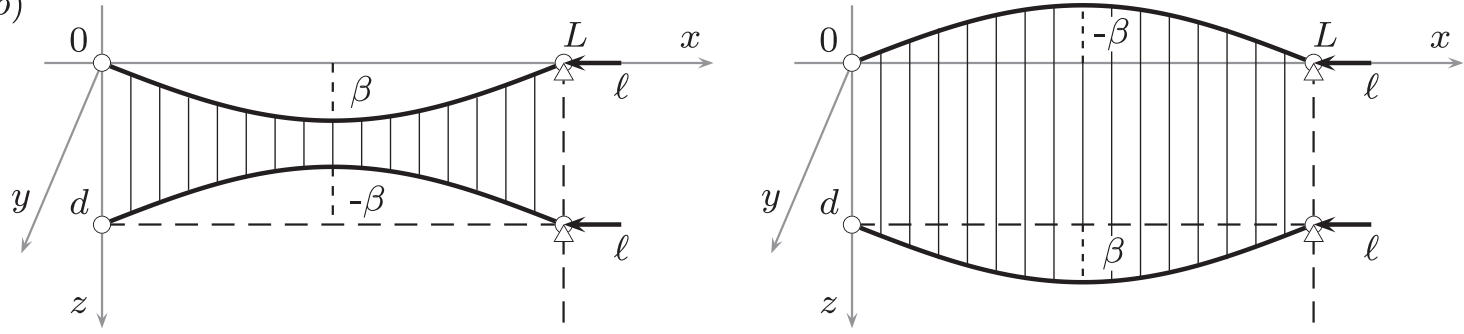

Fig. 7. Symmetrical in-phase (a) and out-of-phase (b) static solutions $(n=1)$.
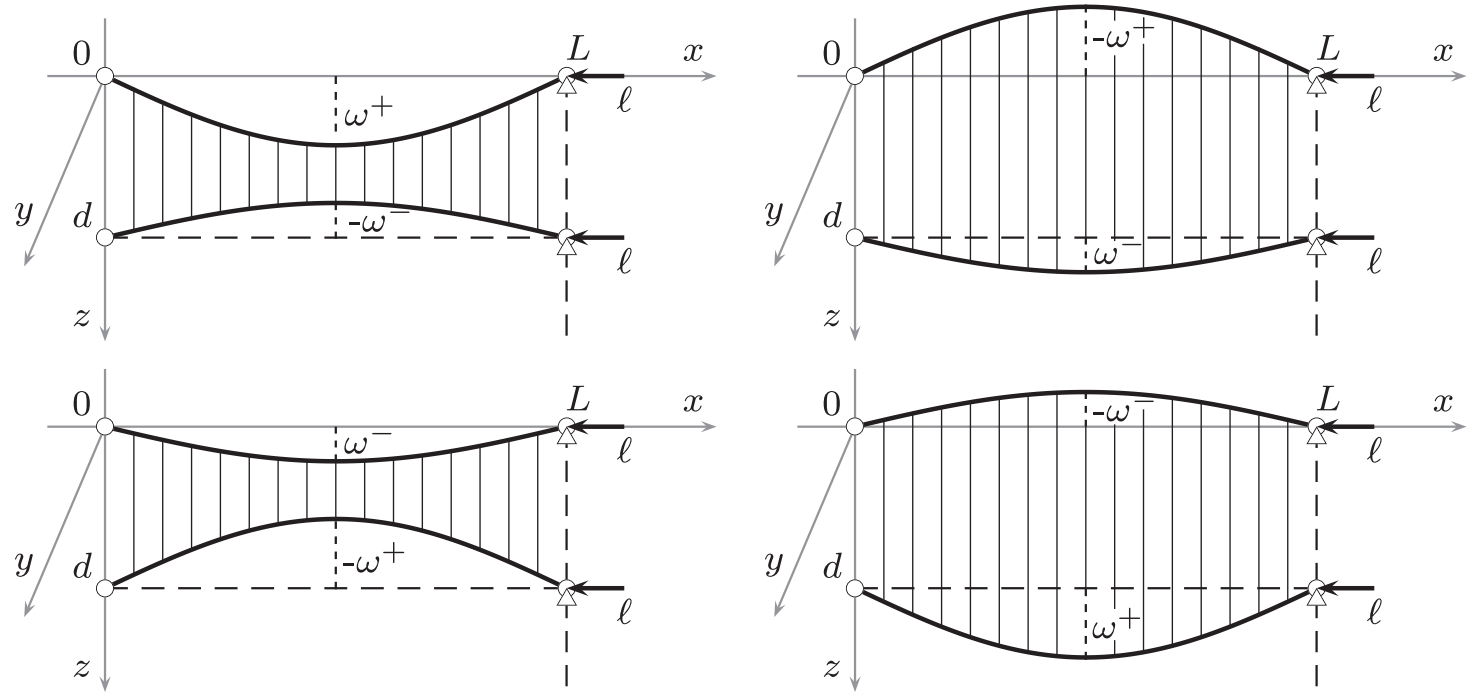

Fig. 8. Asymmetrical out-of-phase static solutions $(n=1)$.

$\pm \xi_{n}^{-1}-\sigma_{n}= \pm \xi_{m}^{-1}-\sigma_{m}$,

which leads to the following four occurrences:

(a) $\xi_{n}^{-1}-\sigma_{n}=\xi_{m}^{-1}-\sigma_{m}$, so that $\lambda_{n}=\lambda_{m}$ which contradicts the assumption $m>n$ and then no bimodal solution exists;

(b) $\xi_{n}^{-1}+\sigma_{n}=\xi_{m}^{-1}+\sigma_{m}$, from which it follows $\kappa=\kappa_{m n}^{\dagger}$ and then $\nu_{n}(\kappa)=\nu_{m}(\kappa)$. If this is the case, (38) takes the form

$\left\{\begin{array}{l}b_{n}=-a_{n}, \\ d_{m}=-c_{m}, \\ \left.a_{n}^{2}+\zeta_{m n} c_{m}^{2}=-2\left(\ell+\mu_{m}+\mu_{n}\right)\right) / L \gamma \sqrt{\lambda_{n}}, \\ \left.b_{n}^{2}+\zeta_{m n} d_{m}^{2}=-2\left(\ell+\mu_{m}+\mu_{n}\right)\right) / L \gamma \sqrt{\lambda_{n}},\end{array}\right.$

where $\zeta_{m n}=\sqrt{\lambda_{m} / \lambda_{n}}>1$, and admits real solutions provided that

$\ell<-\left(\mu_{m}+\mu_{n}\right)=-\nu_{n}\left(\kappa_{m n}^{\dagger}\right)$.

Letting $a_{n}=-b_{n}=U, c_{m}=-d_{m}=V$ and

$\rho_{\uparrow}^{2}(\ell)=-\frac{2 \delta}{L \gamma \sqrt{\lambda_{n}}}\left(\ell+\mu_{m}+\mu_{n}\right)$, we obtain the following (elliptic) relation between the amplitudes

$U^{2}+\zeta_{m n} V^{2}=\rho_{\uparrow}^{2}(\ell)$.

Accordingly, in this case there exist infinitely many out-ofphase solutions;

(c) $\xi_{n}^{-1}-\sigma_{n}=-\xi_{m}^{-1}-\sigma_{m}$, from which it follows $\kappa=\delta\left(\sqrt{\lambda_{n} \lambda_{m}}-\lambda_{m}\right) / 2$ : since $\kappa$ must be positive, its expression contradicts the assumption $m>n$ and then no bimodal solution exists;

(d) $\xi_{n}^{-1}+\sigma_{n}=-\xi_{m}^{-1}+\sigma_{m}$, from which it follows $\kappa=\kappa_{m n}^{\star}$ and then $\nu_{n}(\kappa)=\mu_{m}$. If this is the case, (38) takes the form

$\left\{\begin{array}{l}b_{n}=-a_{n}, \\ d_{m}=c_{m}, \\ a_{n}^{2}+\zeta_{m n} c_{m}^{2}=-2\left(\ell+\mu_{m}\right) / L \gamma \sqrt{\lambda_{n}}, \\ b_{n}^{2}+\zeta_{m n} d_{m}^{2}=-2\left(\ell+\mu_{m}\right) / L \gamma \sqrt{\lambda_{n}}\end{array}\right.$

and admits real solutions provided that

$\ell<-\mu_{m}=-\nu_{n}\left(\kappa_{m n}^{\star}\right)$. 
Letting $a_{n}=-b_{n}=U, c_{m}=d_{m}=V$ and

$\rho_{\star}^{2}(\ell)=-\frac{2 \delta}{L \gamma \sqrt{\lambda_{n}}}\left(\ell+\mu_{m}\right)$,

we obtain the following (elliptic) relation between the amplitudes

$U^{2}+\zeta_{m n} V^{2}=\rho_{\star}^{2}(\ell)$.

Accordingly, in this case there exist infinitely many asymmetric solutions.

\section{A.3. Proof of Theorem 3}

To prove this theorem, first we establish a preliminary result. Letting

$x=\xi_{n}\left(\sigma_{n}+X_{1}\right), \quad y=\xi_{n}\left(\sigma_{n}+X_{2}\right)$,

$w=\xi_{m}\left(\sigma_{m}+X_{1}\right), \quad z=\xi_{m}\left(\sigma_{m}+X_{2}\right)$,

we obtain

$\zeta_{m n} x-w=\zeta_{m n} y-z=\xi_{m}\left(\sigma_{n}-\sigma_{m}\right)=\left(\zeta_{m n}-1\right)\left(1-2 \kappa_{m n}^{\dagger} / \kappa\right)$.

From these relations and (37) we obtain the system

$\left\{\begin{array}{l}x y=1, \\ w z=1, \\ \zeta_{m n} x-w=\left(\zeta_{m n}-1\right)\left(1-2 \kappa_{m n}^{\dagger} / \kappa\right), \\ \zeta_{m n} y-z=\left(\zeta_{m n}-1\right)\left(1-2 \kappa_{m n}^{\dagger} / \kappa\right) .\end{array}\right.$

We stress that the coefficients involved in this representation are independent of $L, \gamma$ and, especially, on $\ell$.

Lemma 9. Let $m>n$ be given, if and only if either $\kappa>2 \kappa_{m n}^{\dagger}$ or $0<\kappa<\kappa_{m n}^{\star}$ or $\kappa^{\dagger}<\kappa<2 \kappa_{m n}^{\dagger}$ system (41) has exactly four distinct and partially symmetric solutions

$\left(x^{*}, y^{*}, w^{*}, z^{*}\right), \quad\left(x^{*}, y^{*}, z^{*}, w^{*}\right), \quad\left(y^{*}, x^{*}, w^{*}, z^{*}\right), \quad\left(y^{*}, x^{*}, z^{*}, w^{*}\right)$,

where $x^{*}, y^{*}, z^{*}, w^{*}$ are defined as follows:

$\chi^{*}(\kappa)=\frac{1}{2}\left(\phi_{m n}(\kappa)+\sqrt{\phi_{m n}^{2}(\kappa)-4}\right)$,

$y^{*}(\kappa)=\frac{1}{2}\left(\phi_{m n}(\kappa)-\sqrt{\phi_{m n}^{2}(\kappa)-4}\right)$,

$W^{*}(\kappa)=\frac{1}{2}\left(\psi_{m n}(\kappa)+\sqrt{\psi_{m n}^{2}(\kappa)-4}\right)$,

$z *(\kappa)=\frac{1}{2}\left(\psi_{m n}(\kappa)-\sqrt{\psi_{m n}^{2}(\kappa)-4}\right)$,

where $\phi$ and $\psi$ are given in (15) and (16), respectively.

When $\kappa=\kappa_{m n}^{\dagger}$ and $\kappa=\kappa_{m n}^{\star}$ all four solutions coincide and the system reduces to that of the equal-energy case. In particular:

- if $\kappa=\kappa_{m n}^{\dagger}$ then $x^{*}=y^{*}=w^{*}=z^{*}=-1$;

- if $\kappa=\kappa_{m n}^{\star}$ then $x^{*}=y^{*}=-1$ and $w^{*}=z^{*}=1$.

Proof. After simple manipulations, from (41) we obtain two uncoupled subsystems

$\left\{\begin{array}{l}x y=1, \\ x+y=\phi_{m n}(\kappa),\end{array} \quad\left\{\begin{array}{l}w z=1, \\ w+z=\psi_{m n}(\kappa) .\end{array}\right.\right.$

If there exist, solutions to (42) have the form of symmetric pairs parametrized by $\kappa$,

$P_{1}:(x, y)=\left(x^{*}(\kappa), y^{*}(\kappa)\right), \quad P_{2}:(x, y)=\left(y *(\kappa), x^{*}(\kappa)\right)$,

$Q_{1}:(w, z)=\left(W^{*}(\kappa), z^{*}(\kappa)\right), \quad Q_{2}:(w, z)=\left(z^{*}(\kappa), W^{*}(\kappa)\right)$,

where

$$
\begin{aligned}
x^{*}(\kappa)= & \frac{1}{2}\left(\phi_{m n}(\kappa)+\sqrt{\phi_{m n}^{2}(\kappa)-4}\right), \quad y *(\kappa) \\
& =\frac{1}{2}\left(\phi_{m n}(\kappa)-\sqrt{\phi_{m n}^{2}(\kappa)-4}\right), \\
W^{*}(\kappa)= & \frac{1}{2}\left(\psi_{m n}(\kappa)+\sqrt{\psi_{m n}^{2}(\kappa)-4}\right), \quad z^{*}(\kappa) \\
= & \frac{1}{2}\left(\psi_{m n}(\kappa)-\sqrt{\psi_{m n}^{2}(\kappa)-4}\right),
\end{aligned}
$$

and then they are ordered so that

$\chi^{*}(\kappa) \geq y^{*}(\kappa), \quad W^{*}(\kappa) \geq Z^{*}(\kappa)$.

These pairs give the four solutions of the problem and represent the coordinate pairs of the intersection points between a rectangular hyperbola (either $x y=1$ or $w z=1$ ) and a straight line (either $x+y=\phi_{m n}(\kappa)$ or $\left.w+z=\psi_{m n}(\kappa)\right)$. Accordingly, real values $x^{*}(\kappa)$, $y^{*}(\kappa)$ and $w^{*}(\kappa), z^{*}(\kappa)$ do exist provided that either $\phi_{m n}(\kappa) \geq 2$ or $\phi_{m n}(\kappa) \leq-2$, and $\psi_{m n}(\kappa) \geq 2$ or $\psi_{m n}(\kappa) \leq-2$, respectively.

- When $\kappa>2 \kappa_{m n}^{\dagger}$, then $\psi_{m n}(\kappa)>\phi_{m n}(\kappa)>2$. Solutions to (42) are positive pairs. In addition, $W^{*}(\kappa)>X^{*}(\kappa)>1>y^{*}(\kappa)>Z^{*}(\kappa)>0$ (see Fig. 9).

- When $\kappa_{m n}^{\dagger} \leq \kappa<2 \kappa_{m n}^{\dagger}$ then $\psi_{m n}(\kappa) \leq \phi_{m n}(\kappa) \leq-2$, the equalities occurring at $\kappa=\kappa_{m n}^{\dagger}$. In this case solutions to (42) are negative pairs and $z^{*}(\kappa) \leq y^{*}(\kappa) \leq-1 \leq X^{*}(\kappa) \leq W^{*}(\kappa)<0$, the equalities occurring at $\kappa=\kappa_{m n}^{\dagger}$ (see Fig. 10).

- When $0<\kappa \leq \kappa_{m n}^{\star}$ then $\phi_{m n}(\kappa) \leq-2$, but $\psi_{m n}(\kappa) \geq 2$, the equalities occurring at $\kappa=\kappa_{m n}^{\star}$. In this case $(42)_{1}$ is solved by negative pairs, whereas (42) 2 has positive solutions and $y^{*}(\kappa) \leq-1 \leq X^{*}(\kappa)<0<Z^{*}(\kappa) \leq 1 \leq W^{*}(\kappa)$, the equalities occurring at $\kappa=\kappa_{m n}^{\star}$ (see Fig. 11).

Remark 3. The case $X_{1}^{*}=X_{2}^{*}$ is recovered if and only if $x^{*}=y^{*}$ and $W^{*}=Z^{*}$. This occurs either when $\kappa=\kappa_{m n}^{\star}$ or $\kappa_{m n}^{\dagger}$. In the former case $\phi_{m n}\left(\kappa_{m n}^{\star}\right)=-2$ and $\psi_{m n}\left(\kappa_{m n}^{\star}\right)=2$, so that $x^{*}\left(\kappa_{m n}^{\star}\right)=y^{*}\left(\kappa_{m n}^{\star}\right)=-1$ and $w^{*}\left(\kappa_{m n}^{\star}\right)=Z^{*}\left(\kappa_{m n}^{\star}\right)=1$. In the latter, $\phi_{m n}\left(\kappa_{m n}^{\dagger}\right)=\psi_{m n}\left(\kappa_{m n}^{\dagger}\right)=-2$, so that $x^{*}\left(\kappa_{m n}^{\dagger}\right)=y^{*}\left(\kappa_{m n}^{\dagger}\right)=W^{*}\left(\kappa_{m n}^{\dagger}\right)=Z^{*}\left(\kappa_{m n}^{\dagger}\right)=-1$, in agreement with Theorem 2 .

Remark 4. The positivity of the norms, that is $X_{1}^{*}>0$ and $X_{2}^{*}>0$, can be established from (40) in dependence of the values of $\kappa$ and $\ell$,

$$
\begin{aligned}
X_{1}^{*} & =-\frac{2}{L \gamma}\left(\mu_{n}+\kappa \frac{1-x^{*}(\kappa)}{\sqrt{\lambda_{n}}}+\ell\right)=-\frac{2}{L \gamma}\left(\mu_{m}+\kappa \frac{1-W^{*}(\kappa)}{\sqrt{\lambda_{m}}}+\ell\right) \\
& >0, \\
X_{2}^{*} & =-\frac{2}{L \gamma}\left(\mu_{n}+\kappa \frac{1-y^{*}(\kappa)}{\sqrt{\lambda_{n}}}+\ell\right)=-\frac{2}{L \gamma}\left(\mu_{m}+\kappa \frac{1-z^{*}(\kappa)}{\sqrt{\lambda_{m}}}+\ell\right) \\
& >0 .
\end{aligned}
$$

Since $y^{*}(\kappa) \leq x^{*}(\kappa)$ and $z^{*}(\kappa) \leq W^{*}(\kappa)$ for all $\kappa$, these conditions are both satisfied provided that $\ell<\ell^{*}(\kappa)$, where

$\ell *(\kappa)=-\mu_{n}-\kappa \frac{1-y^{*}(\kappa)}{\sqrt{\lambda_{n}}}=-\mu_{m}-\kappa \frac{1-Z^{*}(\kappa)}{\sqrt{\lambda_{m}}}$

or alternately

$\ell^{*}(\kappa)=-\nu_{n}(\kappa)+\kappa \frac{1+y^{*}(\kappa)}{\sqrt{\lambda_{n}}}=-\nu_{m}(\kappa)+\kappa \frac{1+z^{*}(\kappa)}{\sqrt{\lambda_{m}}}$.

We note that $\ell^{*}\left(\kappa^{\dagger}\right)=-\nu_{n}\left(\kappa^{\dagger}\right)=-\mu_{m}-\mu_{n} \quad$ and $\ell *\left(\kappa^{\star}\right)=-\nu_{n}\left(\kappa^{\star}\right)=-\mu_{m}$.

According to the previous analysis, from (38) the system involving the unknown amplitudes takes the form 


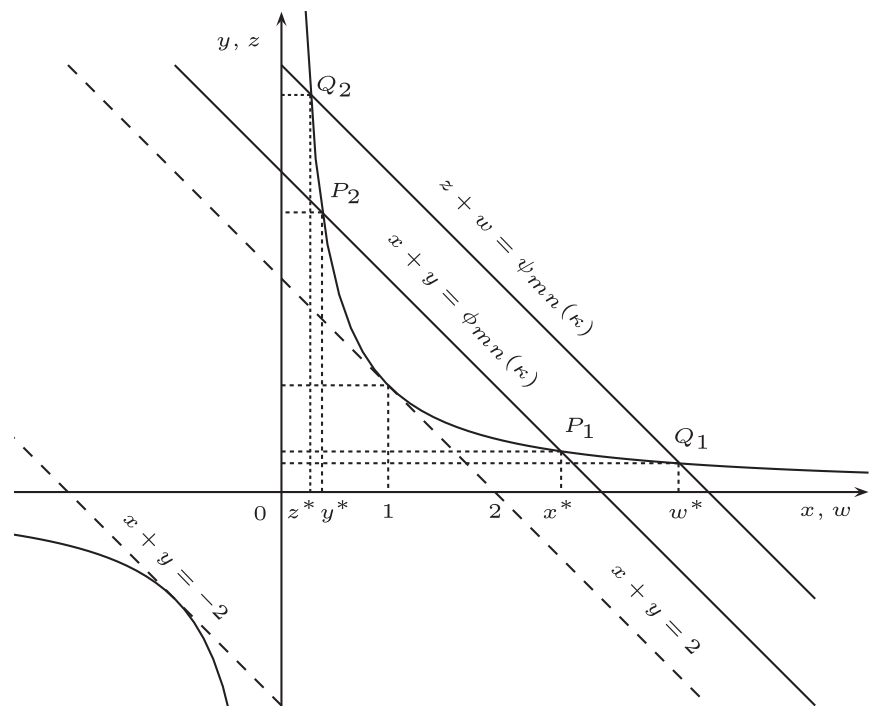

Fig. 9. The graphic solution of subsystems (42) when $\kappa>2 \kappa^{\dagger}$.

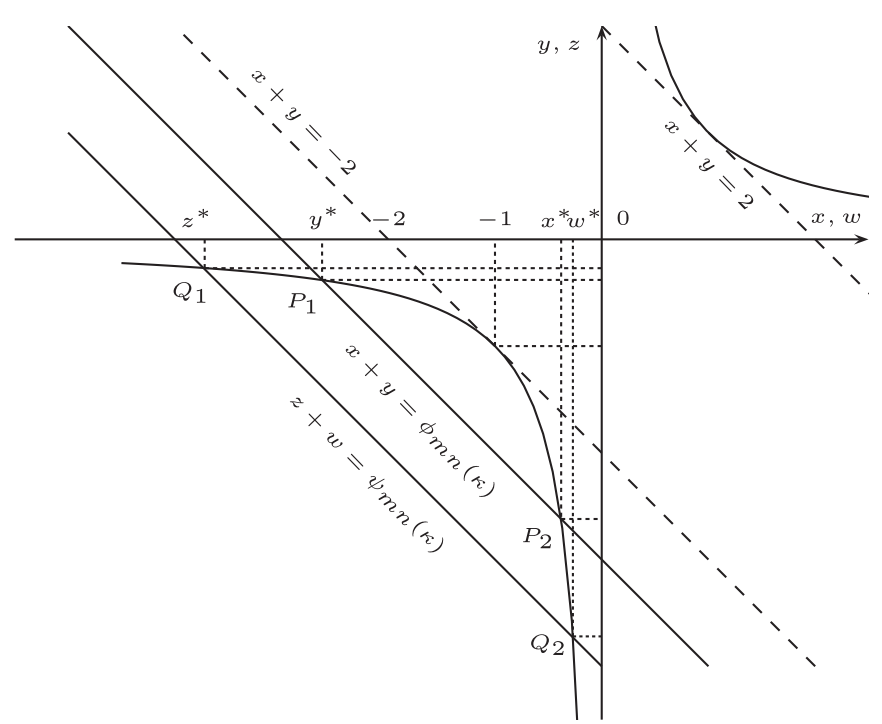

Fig. 10. The graphic solution of subsystems (42) when $\kappa^{\dagger} \leq \kappa<2 \kappa^{\dagger}$.

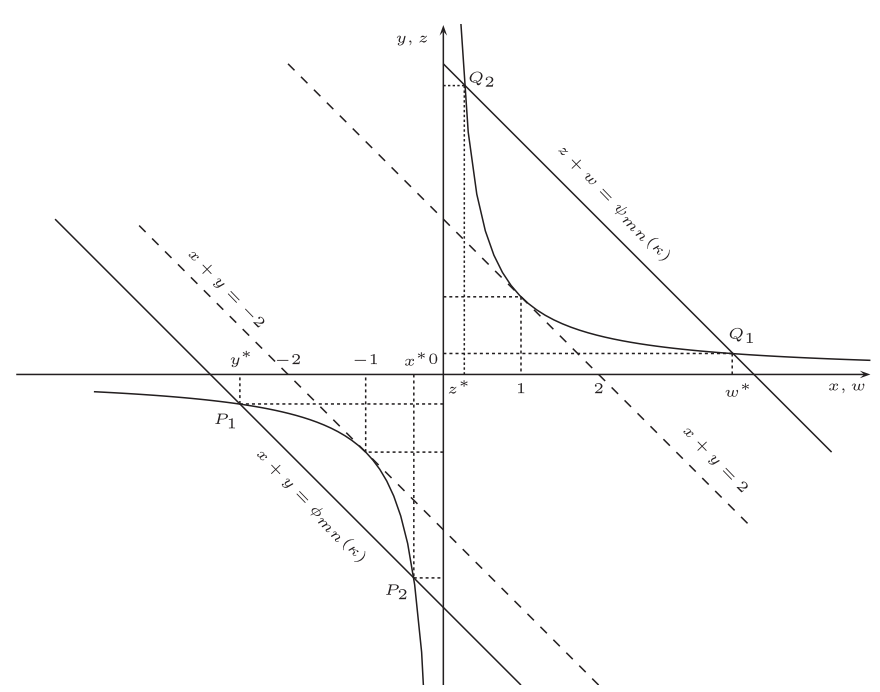

Fig. 11. The graphic solution of subsystems (42) when $0<\kappa \leq \kappa^{\star}$. $\left\{\begin{array}{l}b_{n}=\chi^{*}(\kappa) a_{n}, \\ d_{m}=W^{*}(\kappa) c_{m}, \\ a_{n}^{2}+\zeta_{m n} c_{m}^{2}=\frac{2}{L \gamma \sqrt{\lambda_{n}}}\left(\ell^{*}(\kappa)-\ell+\kappa \frac{\chi^{*}(\kappa)-y^{*}(\kappa)}{\sqrt{\lambda_{n}}}\right), \\ b_{n}^{2}+\zeta_{m n} d_{m}^{2}=\frac{2}{L \gamma \sqrt{\lambda_{n}}}\left(\ell^{*}(\kappa)-\ell\right) .\end{array}\right.$

Assuming $\ell<\ell^{*}$, from (43) we finally obtain

$\left\{\begin{array}{l}b_{n}=x^{*} a_{n}, \\ d_{m}=w^{*} c_{m}, \\ a_{n}^{2}+\zeta_{m n} c_{m}^{2}=\rho^{2}+r^{2}, \\ x^{2} a_{n}^{2}+\zeta_{m n} w *^{2} c_{m}^{2}=\rho^{2},\end{array}\right.$

where

$r^{2}(\kappa)=\frac{2 \kappa}{L \gamma \lambda_{n}}\left(X^{*}(\kappa)-y^{*}(\kappa)\right), \quad \rho^{2}(\kappa, \ell)=\frac{2}{L \gamma \sqrt{\lambda_{n}}}(\ell *(\kappa)-\ell)$.

In particular, $\rho\left(\kappa^{\dagger}, \ell\right)=\rho_{\dagger}(\ell), \rho\left(\kappa^{\star}, \ell\right)=\rho_{\star}(\ell)$ and $r\left(\kappa^{\dagger}\right)=r\left(\kappa^{\star}\right)=0$. In order to find amplitudes of the bimodal solutions we are led to solve the system

$\left\{\begin{array}{l}X^{2}+Y^{2}=\rho^{2}+r^{2}, \\ x^{2} X^{2}+w^{2} Y^{2}=\rho^{2},\end{array}\right.$

where $X=a_{n}, Y=\sqrt{\zeta_{m n}} c_{m}$. Since it represents the intersection between a circle and an ellipse, four real solutions exist if and only if the radius of the circle has an intermediate value between major and minor semi-axes of the ellipse. If this is the case, the intersection points are

$P_{1}:(X, Y)=\left(X^{*}, Y^{*}\right), \quad P_{2}:(X, Y)=\left(-X^{*}, Y^{*}\right)$,

$P_{3}:(X, Y)=\left(X^{*},-Y^{*}\right), \quad P_{4}:(X, Y)=\left(-X^{*},-Y^{*}\right)$.

Accordingly, the amplitudes of the bimodal solutions are

$\left\{\begin{array}{l}a_{n}=X^{*}, \\ b_{n}=X^{*}(\kappa) X^{*}, \\ c_{m}=Y^{*} / \sqrt{\zeta_{m n}}, \\ d_{m}=w^{*}(\kappa) Y^{*} \mid \sqrt{\zeta_{m n}},\end{array} \quad\left\{\begin{array}{l}a_{n}=-X^{*}, \\ b_{n}=-X^{*}(\kappa) X^{*}, \\ c_{m}=Y^{*} \mid \sqrt{\zeta_{m n},} \\ d_{m}=w^{*}(\kappa) Y^{*} / \sqrt{\zeta_{m n}},\end{array}\right.\right.$

$\left\{\begin{array}{l}a_{n}=X^{*}, \\ b_{n}=X^{*}(\kappa) X^{*}, \\ c_{m}=-Y^{*} \mid \sqrt{\zeta_{m n}}, \\ d_{m}=-W^{*}(\kappa) Y^{*} \mid \sqrt{\zeta_{m n}},\end{array} \quad\left\{\begin{array}{l}a_{n}=-X^{*}, \\ b_{n}=-X^{*}(\kappa) X^{*}, \\ c_{m}=-Y^{*} \mid \sqrt{\zeta_{m n}}, \\ d_{m}=-w^{*}(\kappa) Y^{*} \mid \sqrt{\zeta_{m n}} .\end{array}\right.\right.$

In order to establish necessary and sufficient conditions for the existence of such solutions, we separately discuss three cases.

- When $\kappa>2 \kappa_{m n}^{\dagger}$, then $0<y^{*}(\kappa)<1<x^{*}(\kappa)<W^{*}(\kappa)$ and

$\frac{1}{W^{2}} \leq \frac{1}{\chi^{2}} \leq 1 \leq 1+\frac{r^{2}}{\rho^{2}}$.

As a consequence,

$\frac{\rho^{2}}{W^{2}} \leq \frac{\rho^{2}}{x^{2}} \leq \rho^{2}+r^{2}$

and then no solution exists.

- When $\kappa_{m n}^{\dagger} \leq \kappa<2 \kappa_{m n}^{\dagger}$, then $y^{*}(\kappa) \leq-1 \leq \chi^{*}(\kappa) \leq W^{*}(\kappa)<0$. As a consequence, the condition to have intersections reads

$\frac{\rho^{2}}{\boldsymbol{X}^{2}} \leq \rho^{2}+r^{2} \leq \frac{\rho^{2}}{\boldsymbol{W}^{2}}$

and implies the following conditions for $\ell$ : 
$0<\frac{\ell *-\ell}{x^{2}} \leq \ell *-\ell+\frac{\kappa}{\sqrt{\lambda_{n}}}\left(x^{*}-y^{*}\right) \leq \frac{\ell *-\ell}{w^{*}}$.

From the left inequality we obtain $\ell \geq \ell_{1}^{*}$, where $\left(y^{*}=1 / x^{*}\right)$

$\ell_{1}^{*}=\ell^{*}-\frac{\kappa}{\sqrt{\lambda_{n}}} \frac{\chi^{*}\left(\chi^{*}-y^{*}\right)}{1-\chi^{*}}=\ell^{*}+\frac{\kappa}{\sqrt{\lambda_{n}}} X^{*}(\kappa)$,

and then

$\ell_{1}^{*}(\kappa)=-\nu_{n}(\kappa)+\kappa \frac{1+\phi_{m n}(\kappa)}{\sqrt{\lambda_{n}}}$.

From the right inequality and using $(41)_{3}$ we have $\ell \leq \ell_{2}^{*}$, where $\left(Z^{*}=1 / w^{*}\right)$

$\ell_{2}^{*}=\ell^{*}+\frac{\kappa}{\sqrt{\lambda_{m}}} \frac{W^{*}\left(Z^{*}-W^{*}\right)}{1-W^{2}}=\ell^{*}+\frac{\kappa}{\sqrt{\lambda_{m}}} W^{*}(\kappa)<\ell^{*}$

and then

$\ell_{2}^{*}(\kappa)=-\nu_{m}(\kappa)+\kappa \frac{1+\psi_{m n}(\kappa)}{\sqrt{\lambda_{m}}}$.

Summarizing, solutions exist if and only if $\ell_{1}^{*} \leq \ell \leq \ell_{2}^{*}$.

- When $0<\kappa \leq \kappa_{m n}^{\star}$, then $y^{*}(\kappa)<-1<x^{*}(\kappa)<0<1<W^{*}(\kappa)$, which implies

$\frac{\rho^{2}}{W^{2}} \leq \rho^{2} \leq \rho^{2}+r^{2}$.

As a consequence, the condition to have intersections reduces to

$\rho^{2}+r^{2} \leq \frac{\rho^{2}}{\chi^{2}}$

and the following condition for $\ell$ follows:

$\ell *-\ell+\frac{\kappa}{\sqrt{\lambda_{n}}}\left(x^{*}-y^{*}\right) \leq \frac{\ell^{*}-\ell}{x^{2}}$,

from which we obtain $\ell \leq \ell_{1}^{*}$.

We finally observe that

$\ell_{1}^{*}(\kappa)=-\mu_{n}+\kappa \frac{\phi_{m n}(\kappa)-1}{\sqrt{\lambda_{n}}}$

and

$\lim _{\kappa \rightarrow \kappa_{m}^{\dagger} n} \ell_{1}^{*}(\kappa)=-\mu_{n}-\mu_{m}-\frac{\kappa_{m n}^{\dagger}}{\sqrt{\lambda_{n}}}=-\mu_{n}-\frac{3}{2} \mu_{m}$,

$\lim _{\kappa \rightarrow \kappa m n} \ell_{2}^{*}(\kappa)=-\mu_{m}-\mu_{n}-\frac{\kappa_{m n}^{\dagger}}{\sqrt{\lambda_{m}}}=-\mu_{m}-\frac{3}{2} \mu_{n}$,

$\lim _{\kappa \rightarrow \kappa_{m n}^{\star}} \ell_{1}^{*}(\kappa)=-\mu_{n}-\frac{\kappa_{m n}^{\star}}{\sqrt{\lambda_{n}}}=-\frac{1}{2}\left(\mu_{n}+\mu_{m}\right)$.

\section{References}

[1] A.V. Babin, M.I. Vishik, Attractors of Evolution Equations, North-Holland, Amsterdam, 1992.

[2] J.M. Ball, Initial-boundary value problems for an extensible beam, J. Math. Anal. Appl. 42 (1973) 61-90.

[3] J.M. Ball, Stability theory for an extensible beam, J. Differ. Equ. 14 (1973) 399-418.
[4] R. Benito, S. Sridharan, Mode interaction in thin-walled structural members, J. Struct. Mech. 12 (1984) 517-542.

[5] I. Bochicchio, C. Giorgi, E. Vuk, Steady states analysis and exponential stability of an extensible thermoelastic system, Commun. SIMAI Congr. 3 (2009) 232-243, ISSN 1827-9015.

[6] I. Bochicchio, C. Giorgi, E. Vuk, Long-term damped dynamics of the extensible suspension bridge, Int. J. Differ. Equ. 2010 (2010) 19.

[7] I. Bochicchio, E. Vuk, Longtime behavior of an extensible elastic beam on a viscoelastic foundation, Math. Comput. Modell. 51 (2010) 833-846.

[8] I. Bochicchio, E. Vuk, Longtime behavior for oscillations of an extensible viscoelastic beam with elastic external supply, Int. J. Pure Appl. Math. 1 (2010) 61-76.

[9] I. Bochicchio, C. Giorgi, E. Vuk, Long-term dynamics of the coupled suspension bridge system, Math. Models Methods Appl. Sci. 22 (2012) 1250021 (22 pp.).

[10] I. Bochicchio, C. Giorgi, E. Vuk, Asymptotic dynamics of nonlinear coupled suspension bridge equations, J. Math. Anal. Appl. 402 (2013) 319-333.

[11] B. Budiansky, Theory of buckling and postbuckling behaviour of elastic structures, in: Chia-Shun Yih (Ed.), Advances in Applied Mechanics, vol. 14, Academic Press, New York, USA, 1974, pp. 1-65.

[12] M. Conti, V. Pata, Weakly dissipative semilinear equations of viscoelasticity, Commun. Pure Appl. Anal. 4 (2005) 705-720.

[13] M. Conti, V. Pata, M. Squassina, Singular limit of differential systems with memory, Indiana Univ. Math. J. 55 (2006) 169-216.

[14] M. Coti Zelati, Global and exponential attractors for the singularly perturbed extensible beam, Discrete Contin. Dyn. Syst. 25 (2009) 1041-1060.

[15] M. Coti Zelati, C. Giorgi, V. Pata, Steady states of the hinged extensible beam with external load, Math. Models Methods Appl. Sci. 20 (2010) 43-58.

[16] F. Dell'Oro, C. Giorgi, V. Pata, Steady states of elastically-coupled extensible double-beam systems, Quad. Sem. Mat. Brescia XX (2015), arXiv:1607.04021.

[17] A. Dorfmann, R.W. Ogden, Nonlinear electroelastostatics: incremental equations and stability, Int. J. Eng. Sci. 48 (2010) 1-14.

[18] C. Giorgi, M.G. Naso, Modeling and steady states analysis of the extensible thermoelastic beam, Math. Comput. Model. 53 (2011) 896-908.

[19] C. Giorgi, V. Pata, E. Vuk, On the extensible viscoelastic beam, Nonlinearity 21 (2008) 713-733.

[20] J.K. Hale, Asymptotic Behavior of Dissipative Systems, American Mathematical Society, Providence, 1988.

[21] S.G. Kelly, S. Srinivas, Free vibrations of elastically connected stretched beams, J. Sound Vib. 326 (2009) 883-893.

[22] W. Lacarbonara, Nonlinear Structural Mechanics: Theory, Dynamical Phenomena and Modeling, Springer, New York, 2012.

[23] A. Luongo, On amplitude modulation and localization phenomena in interactive buckling problems, J. Sound Vib. 27 (15) (1991) 1943-1954.

[24] A. Luongo, Mode localization by structural imperfections in one-dimensional continuous systems, J. Sound Vib. 155 (2) (1992) 249-271.

[25] T. Murmu, S. Adhikari, Axial instability of a double-nanobeam-systems, Phys. Lett. A 375 (2011) 601-608.

[26] Z. Oniszczuk, Forced transverse vibrations of an elastically connected complex simply supported double-beam system, J. Sound Vib. 264 (2003) 273-286.

[27] C. Pierre, E.H. Dowell, Localization of vibrations by structural irregularity, J. Sound Vib. 114 (3) (1987) 549-564.

[28] C. Pierre, R.H. Plaut, Curve veering and mode localization in a buckling problem, J. Appl. Math. Phys. 40 (1989) 758-761.

[29] M. Pignataro, A. Luongo, Interactive buckling of an elastically restrained truss structure, Thin-Walled Struct. 19 (2-4) (1994) 197-210.

[30] M. Potier-Ferry, Foundations of elastic postbuckling theory, in: Lecture Notes in Physics, Springer, Berlin, 1987, pp. 1-82.

[31] E.L. Reiss, B.J. Matkowsky, Nonlinear dynamic buckling of a compressed elastic column, Q. Appl. Math. 29 (1971) 245-260.

[32] S. Sridharan, M.A. Ali, Interactive buckling in thin-walled beam columns, ASCE III (EM12) (1985) 1470-1486.

[33] R. Temam, Infinite-dimensional Dynamical Systems in Mechanics and Physics, Springer, New York, 1997.

[34] H. Troger, A. Steindl, Nonlinear Stability and Bifurcation Theory, SpringerVerlag, Wien, New York, 1991.

[35] H.V. Vu, A.M. Ordóñez, B.K. Karnopp, Vibration of a double-beam system, J. Sound Vib. 229 (4) (2000) 807-822.

[36] D.H. Wang, G.F. Wang, Surface effects on the vibration and buckling of doublenanobeam-systems, J. Nanomater. 2011 (2011) 7, Article ID 518706.

[37] S. Woinowsky-Krieger, The effect of an axial force on the vibration of hinged bars, J. Appl. Mech. 17 (1950) 35-36.

[38] Y.Q. Zhang, Y. Lu, G.W. Ma, Effect of compressive axial load on forced transverse vibrations of a double-beam system. Int. J. Mech. Sci. 50 (2008) 299-305.

[39] Y.Q. Zhang, Y. Lu, S.L. Wang, X. Liu, Vibration and buckling of a double-beam system under compressive axial loading, J. Sound Vib. 318 (2008) 341-352. 